\title{
Coalition Logic with Individual, Distributed and Common Knowledge*
}

\author{
Thomas Ågotnes \\ Department of Information Science and Media Studies \\ University of Bergen, PB. 7802, 5020 Bergen, Norway \\ thomas.agotnes@infomedia.uib.no \\ Natasha Alechina \\ School of Computer Science \\ University of Nottingham, Nottingham NG8 1BB, UK \\ nza@cs.nott.ac.uk
}

December 1, 2015

\begin{abstract}
Coalition logic is currently one of the most popular logics for multi-agent systems. While logics combining coalitional and epistemic operators have received considerable attention, completeness results for epistemic extensions of coalition logic have so far been missing. In this paper we provide several such results and proofs. We prove completeness for epistemic coalition logic with common knowledge, with distributed knowledge, and with both common and distributed knowledge, respectively. Furthermore, we completely characterise the complexity of the satisfiability problem for each of the three logics. We also study logics with interaction axioms connecting coalitional ability and knowledge.
\end{abstract}

\section{Introduction}

Coalition Logic $(\mathcal{C L})$ [25] is arguably one of the most popular logics that emerged in multi-agent systems research in recent years. The main construction in coalition logic is of the form $[G] \phi$, where $G$ is a set of agents and $\phi$ a formula, intuitively meaning that $G$ is effective for $\phi$, or that $G$ can make $\phi$ come true no matter what the other agents do. Coalition logic allows reasoning about groups of agents having a winning strategy is one shot games, or in general being able to achieve some outcome in interaction with the environment or other groups of agents.

One of the most studied extensions of logics of coalitional ability such as $\mathcal{C L}$ is adding knowledge operators of the type found in epistemic logic [8, 22,27]: both individual knowledge operators $K_{i}$ where $i$ is an agent, and different types of group knowledge operators $E_{G}, C_{G}$ and $D_{G}$ where $G$ is a finite group of agents, standing for everybody-knows, common knowledge and distributed knowledge, respectively. $E_{G} \phi$ means that every agent in the group $G$ knows that $\phi$, and is definable as a conjunction of statements about individual knowledge. The latter two operators are not definable using individual knowledge modalities. $C_{G} \phi$ means that everybody in $G$ knows that $\phi$, but in addition everybody knows that everybody knows that $\phi$, and everybody knows that everybody knows that everybody knows that $\phi$, etc. $D_{G} \phi$ means that if the agents in $G$ combine their knowledge, they will come to know that $\phi$ (for example, if agent $i$ knows that $\psi$ and agent $j$ knows that $\psi \rightarrow \phi$, then $D_{\{i, j\}} \phi$ holds even if neither $i$ nor $j$ know that $\phi$ ).

Combining coalitional ability operators and epistemic operators in general and group knowledge operators in particular lets us express many potentially interesting properties of multi-agent systems, such as [28]:

${ }^{*}$ A preliminary version of this paper, titled Epistemic Coalition Logic: Completeness and Complexity, was presented at AAMAS 2012. 
- $K_{i} \phi \rightarrow[\{i\}] K_{j} \phi: i$ can communicate her knowledge of $\phi$ to $j$;

- $C_{G} \phi \rightarrow[G] \psi$ : common knowledge in $G$ of $\phi$ is sufficient for $G$ to ensure that $\psi$;

- $[G] \psi \rightarrow D_{G} \phi$ : distributed knowledge in $G$ of $\phi$ is necessary for $G$ to ensure that $\psi$;

- $D_{G} \phi \rightarrow[G] E_{G} \phi: G$ can cooperate to make distributed knowledge explicit.

In this paper we study axiomatisation and complexity of variants of epistemic coalition logic $(\mathcal{E C L})$, extensions of coalition logic with operators for individual knowledge and different combinations of common knowledge and distributed knowledge. The question whether existing axiomatisations of $\mathcal{C L}$ and epistemic logics with group modalities together produce a complete axiomatisation of the corresponding extension has not been answered so far ${ }^{1}$. Here, we show that this is indeed the case. We furthermore completely characterise the computational complexity of the satisfiability problem for these extensions. Note that since $\mathcal{C} \mathcal{L}$ is not a normal modal logic, the results presented in this paper do not follow from general results on products of modal logics, as for example in [9].

In this paper we combine standard coalitional ability operators and standard epistemic operators, i.e., we do not change the original semantics of either type of operator. It is well known $[21,20]$ that there are several interesting variants of "ability" under imperfect knowledge; e.g., being able to achieve something without necessarily knowing it, vs. knowing that one is able to achieve something but not necessarily knowing how, vs. knowing how one can achieve something. As discussed in the literature [21, 20], even in the face of imperfect information all of these variants are potentially interesting, and it is important to be able to distinguish between them; for example, the first variant can be used to identify agents or groups who can make something come about if they get enough information, in contrast with those who cannot no matter how much information they get. While the two variants of "ability" just mentioned can be expressed with combinations of operators with standard semantics $([\{i\}] \phi$ and $K_{i}[\{i\}] \phi$ respectively, in the case of a single agent), in order to be able to express the latter (knowledge of ability "de re"), operators with alternative semantics are needed $[21,26,18,20]$. We do not consider such operators in the current paper. Also, while knowledge of ability "de re" makes sense in (e.g.) $\mathcal{A T} \mathcal{L}$, it is not as relevant for $\mathcal{C} \mathcal{L}$ with standard semantics because the standard semantic models of $\mathcal{C} \mathcal{L}$ abstracts away the notion of action identity that is needed to formalise this concept (this problem can of course be solved by using semantic models with more information about actions, such as the standard models of $\mathcal{A T} \mathcal{L})$. Even though $\mathcal{E} \mathcal{L}$ with standard semantics cannot express knowledge of ability "de re", it can express many other interesting properties (including the examples above as well as the other "variants" of ability under imperfect knowledge). In this paper we axiomatise and study the two first variants of ability mentioned above, in a language with both coalitional and epistemic operators. The first is captured by not requiring any interaction properties between the coalitional and the epistemic operators. Taking the first type of ability as a primitive notion, makes it possible to distinguish between it $([\{i\}] \phi)$ and the second type $\left(K_{i}[\{i\}] \phi\right)$ in the same language. We also axiomatise and study the second type of "ability" as a primitive notion, by adding interaction axioms of the type $[\{i\}] \phi \leftrightarrow K_{i}[\{i\}] \phi$.

The rest of the paper is organised as follows. In the next section we first give a brief review of coalition logic, and how it is extended with epistemic operators. We then, in each of the three following sections, consider basic epistemic coalition logic with individual knowledge operators extended with common knowledge, with distributed knowledge, and with both common and distributed knowledge, respectively. For each of these cases we show a completeness result. The reason that we consider each of these three systems separately, rather than only the most expressive logic with both common and distributed knowledge, is first, that we want to carefully chart the results for different combinations of operators (a common practice, also in epistemic logic), and, second, that separate proofs for the common and distributed knowledge cases are useful for further extensions for logics with only these epistemic operators. In Section 6 we consider the computational complexity of the three systems, and in Section 7 we study systems with interaction axioms. We briefly survey related work in Section 8 and conclude in Section 9.

\footnotetext{
${ }^{1}$ In an unpublished abstract of a talk given at the LOFT workshop in 2004 [12], the authors propose an axiomatisation of $\mathcal{A} \mathcal{T} \mathcal{L}$ (which subsumes $\mathcal{C} \mathcal{L}$ ) with individual knowledge and common knowledge operators. However, a completeness result or proof has not been published (personal communication, Valentin Goranko).
} 


\section{Background}

We will define several extensions of propositional logic, with the primitive connections $\neg$ and $\wedge$, and the usual derived connectives, such as $\phi \vee \psi$ for $\neg(\neg \phi \wedge \neg \psi)$ and $\phi \rightarrow \psi$ for $\neg \phi \vee \psi$, will be used. We will also define a number of Hilbert-style axiomatic systems $S$, and by $\vdash_{S} \phi$ we mean that the formula $\phi$ is derivable in system $S$ (under the standard definition of derivability in axiomatic systems). By an $S$-consistent set of formulas, we mean a set from which a contradiction is not derivable in $S$. By a maximal $S$-consistent set of formulas (in a fixed language) we mean an $S$-consistent set such that no proper superset of it (in this language) is consistent.

\subsection{Coalition Logic}

We give a brief overview of Coalition Logic $(\mathcal{C L})$ [25]. Assume a countable set $\Theta$ of atomic propositions, and a finite non-empty set $N$ of agents. A coalition is a set $G \subseteq N$ of agents. We sometimes abuse notation and write a singleton coalition $\{i\}$ as $i$.

The language of $\mathcal{C} \mathcal{L}$ is defined by the following grammar:

$$
\phi::=p|\neg \phi| \phi \wedge \phi \mid[G] \phi
$$

where $p \in \Theta$ and $G \subseteq N$.

A coalition model is a tuple $M=(S, E, V)$, where

- $S$ is a non-empty set of states;

- $V$ is a valuation function, assigning a set $V(s) \subseteq \Theta$ to each state $s \in S$;

- $E$ assigns a truly playable effectivity function (see below) $E(s)$ over $N$ and $S$ to each state $s \in S$.

An effectivity function [25] over $N$ and a set of states $S$ is a function $E$ that maps any coalition $G \subseteq N$ to a set of sets of states $E(G) \subseteq 2^{S}$. An effectivity function is truly playable [25, 11] iff it satisfies the following conditions (when $X \subseteq S, \bar{X}$ denotes the complement $S \backslash X$ ):

E1 $\forall s \in S \forall G \subseteq N: \emptyset \notin E(s)(G)$ (Liveness)

E2 $\forall s \in S \forall G \subseteq N: S \in E(s)(G)$ (Safety)

$\mathbf{E 3} \forall s \in S \forall X \subseteq S: \bar{X} \notin E(s)(\emptyset) \Rightarrow X \in E(s)(N)(N$-maximality)

E4 $\forall s \in S \forall G \subseteq N \forall X \subseteq Y \subseteq S: X \in E(s)(G) \Rightarrow Y \in E(s)(G)$ (outcome monotonicity)

E5 $\forall s \in S \forall G_{1}, G_{2} \subseteq N \forall X, Y \subseteq S: X \in E(s)\left(G_{1}\right)$ and $Y \in E(s)\left(G_{2}\right) \Rightarrow X \cap Y \in E(s)\left(G_{1} \cup G_{2}\right)$, where $G_{1} \cap G_{2}=\emptyset$ (superadditivity)

E6 $E^{n c}(s)(\emptyset) \neq \emptyset$, where $E^{n c}(s)(\emptyset)$ is the non-monotonic core of the empty coalition, namely

$$
E^{n c}(s)(\emptyset)=\{X \in E(s)(\emptyset): \neg \exists Y(Y \in E(s)(\emptyset) \text { and } Y \subsetneq X)\}
$$

An effectivity function that only satisfies E1-E5 is called playable. On finite domains an effectivity function is playable iff it is truly playable [11], because on finite domains E6 follows from E1-E5.

A $\mathcal{C} \mathcal{L}$ formula is interpreted in a state $s$ in a coalition model $M$ as follows:

$$
\begin{aligned}
& M, s \models p \text { iff } p \in V(s) \\
& M, s \models \neg \phi \text { iff } M, s \not \models \phi \\
& M, s \models\left(\phi_{1} \wedge \phi_{2}\right) \text { iff }\left(M, s \models \phi_{1} \text { and } M, s \models \phi_{2}\right) \\
& M, s \models[G] \phi \text { iff } \phi^{M} \in E(s)(G)
\end{aligned}
$$


where $\phi^{M}=\{t \in S: M, t \models \phi\}$.

The axiomatisation $C L$ of coalition logic consist of the following axioms and rules:

Prop Substitution instances of propositional tautologies

$$
\begin{aligned}
& \text { G1 } \neg[G] \perp \\
& \text { G2 }[G] \top \\
& \text { G3 } \neg[\emptyset] \neg \phi \rightarrow[N] \phi \\
& \text { G4 }[G](\phi \wedge \psi) \rightarrow[G] \psi \\
& \text { G5 }\left[G_{1}\right] \phi \wedge\left[G_{2}\right] \psi \rightarrow\left[G_{1} \cup G_{2}\right](\phi \wedge \psi), \text { if } G_{1} \cap G_{2}=\emptyset \\
& \text { MP } \vdash_{C L} \phi, \phi \rightarrow \psi \Rightarrow \vdash_{C L} \psi \\
& \text { RG } \vdash_{C L} \phi \leftrightarrow \psi \Rightarrow \vdash_{C L}[G] \phi \leftrightarrow[G] \psi
\end{aligned}
$$

$C L$ is sound and complete with respect to all coalition models [25].

The following monotonicity rule is derivable [25], and will be useful later:

Mon $\vdash_{C L} \phi \rightarrow \psi \Rightarrow \vdash_{C L}[G] \phi \rightarrow[G] \psi$

\subsection{Adding Knowledge Operators}

Epistemic extensions of coalition logic were first proposed in $[28]^{2}$. They are obtained by extending the language with epistemic operators, and the models with epistemic accessibility relations.

An epistemic accessibility relation for agent $i$ over a set of states $S$ is a binary equivalence relation $\sim_{i} \subseteq S \times S$. An epistemic coalition model, henceforth often simply called a model, is a tuple

$$
M=\left(S,\left\{\sim_{i}: i \in N\right\}, E, V\right)
$$

where $(S, E, V)$ is a coalition model and $\sim_{i}$ is an epistemic accessibility relation over $S$ for each agent $i$.

Epistemic operators come in two types: individual knowledge operators $K_{i}$, where $i$ is an agent, and group knowledge operators $C_{G}$ and $D_{G}$ where $G$ is a coalition, for expressing common knowledge and distributed knowledge, respectively. Formally, the language of $\mathcal{C L C D}$ (coalition logic with common and distributed knowledge), is defined by extending coalition logic with all of these operators:

$$
\phi::=p|\neg \phi| \phi \wedge \phi|[H] \phi| K_{i} \phi\left|C_{G} \phi\right| D_{G} \phi
$$

where $p \in \Theta, i \in N, H \subseteq N$ and $\emptyset \neq G \subseteq N$. When $G$ is a coalition, we write $E_{G} \phi$ as a shorthand for $\bigwedge_{i \in G} K_{i} \phi$ (everyone in $G$ knows $\phi$ ).

The languages of the logics $\mathcal{C} \mathcal{L}, \mathcal{C} \mathcal{L C}$ and $\mathcal{C} \mathcal{L}$ are the restrictions of this language with no $C_{G}$ and no $D_{G}$ operators, no $D_{G}$ operators, and no $C_{G}$ operators, respectively.

The interpretation of these languages in an (epistemic coalition) model $M$ is defined by adding the following clauses to the definition for $\mathcal{C} \mathcal{L}$ :

$$
\begin{aligned}
& M, s \models K_{i} \phi \text { iff } \forall t \in S,(s, t) \in \sim_{i} \Rightarrow M, t=\phi \\
& M, s \models C_{G} \phi \text { iff } \forall t \in S,(s, t) \in \sim_{G}^{C} \Rightarrow M, t=\phi \\
& M, s \models D_{G} \phi \text { iff } \forall t \in S,(s, t) \in \sim_{G}^{D} \Rightarrow M, t \models \phi
\end{aligned}
$$

where $\sim_{G}^{C}=\left(\bigcup_{i \in G} \sim_{i}\right)^{*}, R^{*}$ denotes the transitive closure of the relation $R$, and $\sim_{G}^{D}=\left(\bigcap_{i \in G} \sim_{i}\right)$. We use $\models \phi$ to denote the fact that $\phi$ is valid, i.e., that $M, s \models \phi$ for all models $M$ and states $s$ in $M$.

\footnotetext{
${ }^{2}$ In that paper for $\mathcal{A T} \mathcal{L} ; \mathcal{C} \mathcal{L}$ is a fragment of $\mathcal{A T} \mathcal{L}$.
} 


\subsubsection{Some Auxiliary Definitions}

The following are some auxiliary concepts that will be useful in the following.

Intuitively, a pseudomodel is like a model except that distributed knowledge is "not quite" the intersection of individual knowledge. Formally, a pseudomodel is a tuple $M=\left(S,\left\{\sim_{i}: i \in N\right\},\left\{R_{G}: \emptyset \neq G \subseteq N\right\}, E, V\right)$ where $\left(S,\left\{\sim_{i}: i \in N\right\}, E, V\right)$ is a model and:

- $R_{G} \subseteq S \times S$ is an equivalence relation for each $G \subseteq N, G \neq \emptyset$

- For any $i \in N, R_{i}=\sim_{i}$

- For any $G, H, G \subseteq H$ implies that $R_{H} \subseteq R_{G}$

The interpretation of a $\mathcal{C L C D}$ formula in a state of a pseudomodel is defined as for a model, except for the case for $D_{G}$ which is interpreted by the $R_{G}$ relation:

$$
M, s \models D_{G} \phi \text { iff } \forall t \in S,(s, t) \in R_{G} \Rightarrow M, t \models \phi
$$

An epistemic model is a model without the $E$ function, i.e., a tuple $\left(S,\left\{\sim_{i}: i \in N\right\}, V\right)$. An epistemic pseudomodel is a pseudomodel without the $E$ function, i.e., a tuple $\left(S,\left\{\sim_{i}: i \in N\right\},\left\{R_{G}: \emptyset \neq G \subseteq N\right\}, V\right)$ (where $R_{G}$ has the properties above). When $M=\left(S,\left\{\sim_{i}: i \in N\right\}, E, V\right)$ is a model or $M=\left(S,\left\{\sim_{i}: i \in\right.\right.$ $\left.N\},\left\{R_{G}: \emptyset \neq G \subseteq N\right\}, E, V\right)$ is a pseudomodel, we refer to $\left(S,\left\{\sim_{i}: i \in N\right\}, V\right)$ as $M$ 's underlying epistemic model.

We refer to (pseudo)models where $E$ does not satisfy the E6 property as playable (pseudo)models (to distinguish them from models that are "truly playable").

We say that a formula $\phi$ is satisfied in a (playable) (pseudo)model $M$, if $M, s \mid=\phi$ for some state $s$ in $M$.

\section{Coalition Logic with Common Knowledge}

In this section we consider the logic $\mathcal{C} \mathcal{L C}$, extending coalition logic with operators for individual knowledge and common knowledge. The axiomatisation $C L C$ is the result of extending $C L$ with the following standard axioms and rules for individual and common knowledge (see, e.g., [8]):

$\mathbf{K} K_{i}(\phi \rightarrow \psi) \rightarrow\left(K_{i} \phi \rightarrow K_{i} \psi\right)$

$\mathbf{T} K_{i} \phi \rightarrow \phi$

$4 K_{i} \phi \rightarrow K_{i} K_{i} \phi$

$5 \neg K_{i} \phi \rightarrow K_{i} \neg K_{i} \phi$

C $C_{G} \phi \rightarrow E_{G}\left(\phi \wedge C_{G} \phi\right)$

$\mathbf{R N} \vdash_{C L C} \phi \Rightarrow \vdash_{C L C} K_{i} \phi$

$\mathbf{R C} \vdash_{C L C} \psi \rightarrow E_{G}(\phi \wedge \psi) \Rightarrow \vdash_{C L C} \psi \rightarrow C_{G} \phi$

It is easy to show that $C L C$ is sound with respect to all models.

Lemma 1 (Soundness) For any $\mathcal{C} \mathcal{L C}$-formula $\phi, \vdash_{C L C} \phi$ implies $\models \phi$. 


\subsection{Completeness}

In the remainder of this section we show that $\mathcal{C} \mathcal{L C}$ also is complete. Before giving all the details, we describe the outline of the proof. We first construct a canonical playable model $M^{c}$, using standard definitions of the canonical epistemic accessibility relations [16] and Pauly's definition of the canonical effectivity functions [25]. There are two potential problems with $M^{c}$ : first, it is not necessarily truly playable (i.e., it is not necessarily a model), and, second, the truth lemma (stating that a formula is true in a state/maximal consistent set of formulas if and only if it is an element of this set) does not necessarily work for the case $C_{G} \phi$. To take care of these problems we filtrate $M^{c}$ through an appropriately defined closure of a given consistent formula, to obtain a finite model $M^{f}$. This is a standard technique for dealing with transitive closure operators such as the Kleene star in $\mathcal{P} \mathcal{D} \mathcal{L}$ [17] and indeed common knowledge. In our case the standard technique must be extended to deal with the effectivity functions. For us the technique has the convenient side effect that playability and true playability coincide (E1-E5 implies E6) on the resulting model, since it is finite. However, it remains to be shown that filtration does not break the playability properties E1-E5, and that $M^{f}$ satisfies the truth lemma for the combined (epistemic-coalitional) language.

Now, to the details.

Theorem 1 Any CLC-consistent formula is satisfied in some model.

Proof We define a canonical playable model $M^{c}=\left(S^{c},\left\{\sim_{i}^{c}: i \in N\right\}, E^{c}, V^{c}\right)$ as follows:

$S^{c}$ is the set of all maximal $C L C$-consistent sets of formulas;

$s \sim_{i}^{c} t$ iff $\left\{\psi: K_{i} \psi \in s\right\}=\left\{\psi: K_{i} \psi \in t\right\} ;$

$X \in E^{c}(s)(G)$ (for $G \neq N$ ) iff there exists $\psi$ such that $\left\{t \in S^{c}: \psi \in t\right\} \subseteq X$ and $[G] \psi \in s$;

$X \in E^{c}(s)(N)$ iff $S^{c} \backslash X \notin E^{c}(s)(\emptyset) ;$

$p \in V^{c}(s)$ iff $p \in s$.

That $\sim_{i}^{c}$ is an equivalence relation is immediate. That $E^{c}(s)$ is playable (satisfies E1-E5) can be shown in exactly the same way as in the completeness proof for $C L$ [25]. The idea behind the model construction of course is that a formula belongs to a state $s$ in a model iff it is true there (the truth lemma). However, the canonical model is in general not guaranteed to satisfy every consistent formula in the $\mathcal{C} \mathcal{L C}$ language; the case of $C_{G}$ in the truth lemma does not necessarily hold. Therefore we are going to transform $M^{c}$ by filtration into a finite model for a given $C L C$ consistent formula $\phi$. Note that since $\phi$ is consistent, it will belong to at least one $s$ in $M^{c}$.

We abbreviate $\left\{s \in S^{c}: \psi \in s\right\}$ as $[\psi]^{c}$. Note that if for any two formulas $\psi_{1}$ and $\psi_{2},\left[\psi_{1}\right]^{c} \subseteq\left[\psi_{2}\right]^{c}$, then $\vdash_{C L C} \psi_{1} \rightarrow \psi_{2}$ (since $S^{c}$ contains all sets of formulas maximally consistent with respect to $C L C$ ).

Let $c l(\phi)$ be the least set such that

- $c l(\phi)$ contains all subformulas of $\phi$,

- $\operatorname{cl}(\phi)$ is closed under single negations, ${ }^{3}$

- $C_{G} \psi \in \operatorname{cl}(\phi) \Rightarrow K_{i} C_{G} \psi \in \operatorname{cl}(\phi)$ for all $i \in G$, and

- $[G] \psi \in \operatorname{cl}(\phi), G \neq \emptyset \Rightarrow C_{G}[G] \psi \in \operatorname{cl}(\phi)$.

We are going to filtrate $M^{c}$ through $c l(\phi)$. The resulting model $M^{f}=\left(S^{f},\left\{\sim_{i}^{f}: i \in N\right\}, E^{f}, V^{f}\right)$ is constructed as follows:

$S^{f}=\left\{[s]_{c l(\phi)}: s \in S^{c}\right\}$ where $[s]_{c l(\phi)}=s \cap c l(\phi)$. We will omit the subscript $c l(\phi)$ in what follows for readability.

$[s] \sim_{i}^{f}[t]$ iff $\left\{\psi: K_{i} \psi \in[s]\right\}=\left\{\psi: K_{i} \psi \in[t]\right\}$.

$V^{f}([s])=\{p: p \in[s]\}$.

\footnotetext{
${ }^{3} \mathrm{~A}$ set $X$ is closed under single negations if for every $\chi \in X$, if $\chi$ is not of the form $\neg \psi$, then $\neg \chi \in X$.
} 


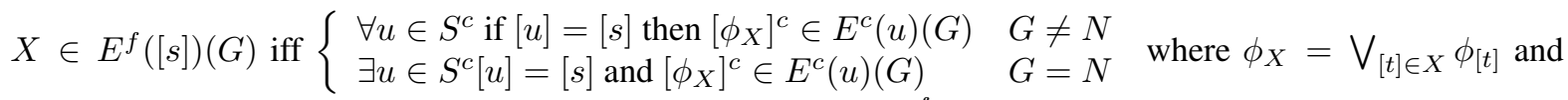
$\phi_{[t]}$ is a conjunction of all formulas in $[t]$. To see that $E^{f}$ is well defined, observe that $[s]=\left[s^{\prime}\right] \Rightarrow$ $E^{f}([s])(G)=E^{f}\left(\left[s^{\prime}\right]\right)(G)$ for any $G$.

We first show some useful properties of the definitions above.

Lemma 2 For any state $u$ in $M^{c}$ and formula $\psi \in \operatorname{cl}(\phi)$,

1. $\left[\bigvee_{[u]: \psi \in[u]} \phi_{[u]}\right]^{c}=[\psi]^{c}$

2. $[\psi]^{c} \in E^{c}(u)(G)$ iff $[G] \psi \in u$, for any $G$

\section{Proof}

1. Assume $S^{f}$ contains $n+k$ states, $\left[t_{1}\right], \ldots,\left[t_{n}\right]$ contain $\psi$ and $\left[s_{1}\right], \ldots,\left[s_{k}\right]$ contain $\neg \psi$. Clearly, $\phi_{\left[t_{1}\right]} \vee$ $\ldots \vee \phi_{\left[t_{n}\right]} \vee \phi_{\left[s_{1}\right]} \ldots \vee \phi_{\left[s_{k}\right]}$ is provably equivalent in $C L C$ to $T$. Consider $\phi_{\left[t_{1}\right]} \vee \ldots \vee \phi_{\left[t_{n}\right]}$. It is provably equivalent to $\left(\psi \wedge \phi_{\left[t_{1}\right]}\right) \vee \ldots \vee\left(\psi \wedge \phi_{\left[t_{n}\right]}\right)$. Since for every $\left[s_{i}\right]$ such that $\neg \psi \in\left[s_{i}\right],\left(\psi \wedge \phi_{\left[s_{i}\right]}\right)$ is provably equivalent to $\perp$,

is provably equivalent to

$$
\left(\psi \wedge \phi_{\left[t_{1}\right]}\right) \vee \ldots \vee\left(\psi \wedge \phi_{\left[t_{n}\right]}\right)
$$

$$
\left(\psi \wedge \phi_{\left[t_{1}\right]}\right) \vee \ldots \vee\left(\psi \wedge \phi_{\left[t_{n}\right]}\right) \vee\left(\psi \wedge \phi_{\left[s_{1}\right]}\right) \vee \ldots \vee\left(\psi \wedge \phi_{\left[s_{k}\right]}\right)
$$

which in turn is provably equivalent to

$$
\psi \wedge\left(\phi_{\left[t_{1}\right]} \vee \ldots \vee \phi_{\left[s_{k}\right]}\right)
$$

which in turn is equivalent to $\psi \wedge \top$ hence to $\psi$.

2. First we consider the case when $G \neq N$. The direction to the left is immediate: if $[G] \psi \in u$ then $[\psi]^{c} \in$ $E^{c}(u)(G)$ by definition. For the other direction assume that $[\psi]^{c} \in E^{c}(u)(G)$, i.e., there is some $\gamma$ such that $[\gamma]^{c} \subseteq[\psi]^{c}$ and $[G] \gamma \in u$. Since $\vdash_{C L C} \gamma \rightarrow \psi$, by the monotonicity rule it follows that $[G] \psi \in u$.

For the case when $G=N$, consider first the direction to the left. Let $[N] \psi \in u$. Then $\neg[\emptyset] \neg \psi \in u$. If there existed a formula $\chi$ such that $[\chi]^{c} \subseteq[\neg \psi]^{c}$ and $[\emptyset] \chi \in u$, we would get, by the monotonicity rule, $[\emptyset] \neg \psi \in u$, which would make $u$ inconsistent; hence no such $\chi$ exists and $[\neg \psi]^{c} \notin E^{c}(u)(\emptyset)$. But then by the definition of $E^{c}$ for $N$ and the fact that $[\neg \psi]^{c}=S^{c} \backslash[\psi]^{c}$, we get $[\psi]^{c} \in E^{c}(u)(N)$.

For the direction to the right, let $[\psi]^{c} \in E^{c}(u)(N)$; then by the definition of $E^{c}$ for $N,[\neg \psi]^{c} \notin E^{c}(u)(\emptyset)$. Then there is no formula $\chi$ such that $[\chi]^{c} \subseteq[\neg \psi]^{c}$ and $[\emptyset] \chi \in u$; in particular, $[\emptyset] \neg \psi \notin u$. This implies that $\neg[\emptyset] \neg \psi \in u$ and $[N] \psi \in u$.

We now prove that for every $\theta \in \operatorname{cl}(\phi), M^{f},[s] \models \theta$ iff $\theta \in[s]$. The proof is by induction on the length of $\theta$ (the length of a formula is the number of symbols required to write down the formula).

case $\theta=p$ trivial

case booleans trivial

case $\theta=K_{i} \psi$ : standard epistemic logic

case $\theta=[G] \psi, G \neq N$

$M^{f},[s] \models[G] \psi$ iff $\psi^{M^{f}} \in E^{f}([s])(G)$ iff $\forall u \in S^{c}$ if $[u]=[s]$ then $\left[\bigvee_{[t] \in \psi^{M^{f}}} \phi_{[t]}\right]^{c} \in E^{c}(u)(G)$ iff (by the inductive hypothesis) $u \in S^{c},[u]=[s] \Rightarrow\left[\bigvee_{\psi \in[t]} \phi_{[t]}\right]^{c} \in E^{c}(u)(G)$ iff (by Lemma 2.1) $\forall u \in S^{c}$, $[u]=[s] \Rightarrow[\psi]^{c} \in E^{c}(u)(G)$ iff (by Lemma 2.2) $\forall u \in S^{c},[u]=[s] \Rightarrow[G] \psi \in u$, which, since $[G] \psi \in \operatorname{cl}(\phi)$, holds iff $[G] \psi \in[s]$. 
case $\theta=[G] \psi, G=N$

$M^{f},[s]=[G] \psi$ iff $\psi^{M^{f}} \in E^{f}([s])(G)$ iff $\exists u \in S^{c}[u]=[s]$ and $\left[\bigvee_{[t] \in \psi^{M}} \phi_{[t]} \in E^{c}(u)(G)\right.$ iff (by the inductive hypothesis) $\exists u \in S^{c}[u]=[s]$ and $[\psi]^{c} \in E^{c}(u)(G)$ iff (by Lemma 2.1) $\exists u \in S^{c}[u]=[s]$ and $[\psi]^{c} \in E^{c}(u)(G)$ iff (by Lemma 2.2) $\exists u \in S^{c}[u]=[s]$ and $[G] \psi \in u$, which, since $[G] \psi \in \operatorname{cl}(\phi)$, holds iff $[G] \psi \in[s]$.

case $\theta=C_{G} \psi$

The proof is similar to that in [29]. First we show that in $M^{f}$, if $C_{G} \psi \in c l(\phi)$, then $C_{G} \psi \in[s]$ iff every state on every $\bigcup_{i \in G} \sim_{i}^{f}$ path from $[s]$ contains $\psi$.

Suppose $C_{G} \psi \in[s]$. The proof is by induction on the length of the path. If the path is of 0 length, then since $C_{G} \psi$ implies $\psi$ in $C L C$ and maximally consistent sets are deductively closed, and by $\psi \in \operatorname{cl}(\phi)$ we have $\psi \in[s]$. We also have $C_{G} \psi \in[s]$ by the assumption.

Inductive hypothesis: if $C_{G} \psi \in[s]$, then every state on every $\bigcup_{i \in G} \sim_{i}^{f}$ path of length $n$ from $[s]$ contains $\psi$ and $C_{G} \psi$. Inductive step: let us prove this for paths of length $n+1$. Suppose we have a path $[s] \sim_{i_{1}}^{f}$ $\left[s_{1}\right] \ldots \sim_{i_{n}}^{f}\left[s_{n}\right] \sim \sim_{i_{n+1}}^{f}\left[s_{n+1}\right]$. By the inductive hypothesis, $\psi, C_{G} \psi \in\left[s_{n}\right]$. Since $s_{n}$ is deductively closed and $K_{i_{n+1}} C_{G} \psi \in \operatorname{cl}(\phi)$, we have $K_{i_{n+1}} C_{G} \psi \in\left[s_{n}\right]$. Since $\left[s_{n}\right] \sim_{i_{n+1}}^{f}\left[s_{n+1}\right]$ and the definition of $\sim_{i_{n+1}}^{f}$, $C_{G} \psi \in\left[s_{n+1}\right]$ and hence since $\psi$ is derivable from $C_{G} \psi$ in $C L C, \psi \in\left[s_{n+1}\right]$.

For the other direction, suppose that every state on every $\bigcup_{i \in G} \sim^{f}$ path from $[s]$ contains $\psi$. Prove that $C_{G} \psi \in[s]$. Let $S_{G, \psi}$ be the set of all $[t]$ such that every state on every $\bigcup_{i \in G} \sim^{f}$ path from $[t]$ contains $\psi$. Note that each $[t]$ is a finite set of formulas so we can write its conjunction $\phi_{[t]}$. Consider a formula

$$
\chi=\bigvee_{[t] \in S_{G, \psi}} \phi_{[t]}
$$

Similarly to [29] it can be proved that $\vdash_{C L C} \phi_{[s]} \rightarrow \chi, \vdash_{C L C} \chi \rightarrow \psi$ and $\vdash_{C L C} \chi \rightarrow E_{G} \chi$. And from that follows that $\vdash_{C L C} \phi_{[s]} \rightarrow C_{G} \psi$ hence $C_{G} \psi \in[s]$.

Now we prove that $M^{f},[s] \models C_{G} \psi$ iff $C_{G} \psi \in[s] . C_{G} \psi \in[s]$ iff every state on every $\bigcup_{i \in G} \sim_{i}^{f}$ path from $[s]$ contains $\psi$ iff for every $[t]$ reachable from $[s]$ by a $\bigcup_{i \in G} \sim_{i}^{f}$ path, $M^{f},[t] \models \psi$ iff $M^{f},[s] \models C_{G} \psi$.

It is obvious that in $M^{f}, \sim_{i}^{f}$ are equivalence relations. So what remains to be proved is that $E^{f}$ satisfies E1-E6. Since $S^{f}$ is finite, it suffices to show E1-E5, which for finite sets of states entail E6.

Proposition $1 M^{f}$ satisfies E1-E5.

\section{Proof}

E1 Note that $\phi_{\emptyset}$ is the empty disjunction, $\perp$.

First, consider the case that $G \neq N$. $\emptyset \in E^{f}([s])(G)$ iff (by definition of $\left.E^{f}\right) \forall u \in S^{c}[u]=[s] \Rightarrow$ $[\perp]^{c} \in E^{c}(u)(G)$ iff $\forall u \in S^{c}[u]=[s] \Rightarrow \emptyset \in E^{c}(u)(G)$. Since $E^{c}$ satisfies E1, $\emptyset \notin E^{c}(s)(G)$, so, $\emptyset \notin E^{f}([s])(G)$.

Second, consider the case that $G=N$. $\emptyset \in E^{f}([s])(G)$ iff $\exists u \in S^{c}[u]=[s]$ and $[\perp]^{c} \in E^{c}(u)(G)$ iff $\exists u \in S^{c}[u]=[s]$ and $\emptyset \in E^{c}(u)(G)$. Since $E^{c}(u)$ satisfies E1 for every $u, \emptyset \notin E^{c}(u)(G)$ for every $u$, so $\emptyset \notin E^{f}([s])(G)$.

E2 First, consider the case that $G \neq N . S^{f} \in E^{f}([s])(G)$ iff $\forall u \in S^{c}[u]=[s] \Rightarrow\left[\bigvee_{[t] \in S^{f}} \phi_{[t]}\right]^{c} \in E^{c}(u)(G)$ iff $\forall u \in S^{c}[u]=[s] \Rightarrow S^{c} \in E^{c}(u)(G)$. Since $E^{c}(u)$ satisfies E2 for every $u, S^{f} \in E^{f}([s])(G)$.

Second, consider the case that $G=N . S^{f} \in E^{f}([s])(G)$ iff $\exists u \in S^{c}[u]=[s]$ and $\left[\bigvee_{[t] \in S^{f}} \phi_{[t]}\right]^{c} \in$ $E^{c}(u)(G)$ iff $\exists u \in S^{c}[u]=[s]$ and $S^{c} \in E^{c}(u)(G)$. Since $E^{c}(s)$ satisfies E2, $S^{f} \in E^{f}([s])(G)$ (take $u=s)$. 
E3 Let $\bar{X} \notin E^{f}([s])(\emptyset)$. Then there is an $u$ such that $[u]=[s]$ and $\left[\phi_{\bar{X}}\right]^{c} \notin E^{c}(u)(\emptyset)$. Note that $\left[\phi_{\bar{X}}\right]^{c}$ is the complement of $\left[\phi_{X}\right]^{c}$, since $\phi_{\bar{X}}=\neg \phi_{X}$. Since $E^{c}(u)$ satisfies E3, this means that there is an $u$ such that $[u]=[s]$ and $\left[\phi_{X}\right]^{c} \in E^{c}(u)(N)$. Hence $X \in E^{f}([s])(N)$ by definition of $E^{f}([s])(G)$ in the case $G=N$.

E4 Let $X \subseteq Y \subseteq S^{f}$ and $X \in E^{f}([s])(G)$. Clearly $\vdash_{C L C} \phi_{X} \rightarrow \phi_{Y}$. Hence $\left[\phi_{X}\right]^{c} \subseteq\left[\phi_{Y}\right]^{c}$. First, consider the case that $G \neq N$. Since $X \in E^{f}([s])(G)$, we have $\left[\phi_{X}\right]^{c} \in E^{c}(u)(G)$ for every $u \in S^{c}$ such that $[u]=[s]$. Since $E^{c}(u)$ satisfies E4, $\left[\phi_{Y}\right]^{c} \in E^{c}(u)(G)$ for every such $u$, so $Y \in E^{f}([s])(G)$.

Second, consider the case that $G=N$. Since $X \in E^{f}([s])(G)$, we have $\left[\phi_{X}\right]^{c} \in E^{c}(u)(G)$ for some $u \in S^{c}$ such that $[u]=[s]$. Since $E^{c}(u)$ satisfies E4, $\left[\phi_{Y}\right]^{c} \in E^{c}(u)(G)$ for some such $u$, so $Y \in E^{f}([s])(G)$.

E5 Let $X \in E^{f}([s])\left(G_{1}\right)$ and $Y \in E^{f}([s])\left(G_{2}\right)$ and $G_{1} \cap G_{2}=\emptyset$.

First, consider the case that $G_{1} \cup G_{2} \neq N$ : for every $u$ such that $[u]=[s],\left[\phi_{X}\right]^{c} \in E^{c}(u)\left(G_{1}\right)$ and $\left[\phi_{Y}\right]^{c} \in E^{c}(u)\left(G_{2}\right)$ and since $E^{c}(u)$ satisfies E5, $\left[\phi_{X}\right]^{c} \cap\left[\phi_{Y}\right]^{c} \in E^{c}(u)\left(G_{1} \cup G_{2}\right)$ for every such $u$. Note that $\left[\phi_{X}\right]^{c} \cap\left[\phi_{Y}\right]^{c}=\left[\bigvee_{[t] \in X \cap Y} \phi_{[t]}\right]^{c}$. Since for every $u$ such that $[u]=[s],\left[\bigvee_{[t] \in X \cap Y} \phi_{[t]}\right]^{c} \in$ $E^{c}(u)\left(G_{1} \cup G_{2}\right)$, we have that $X \cap Y \in E^{f}([s])\left(G_{1} \cup G_{2}\right)$.

Second, consider the case that $G_{1} \cup G_{2}=N$. We consider the three sub-cases:

$G_{1}=N, G_{2}=\emptyset$ : We have that there exists a $u^{\prime}$ such that $\left[u^{\prime}\right]=[s]$ and $\left[\phi_{X}\right]^{c} \in E^{c}\left(u^{\prime}\right)\left(G_{1}\right)$, and that for every $u$ such that $[u]=[s],\left[\phi_{Y}\right]^{c} \in E^{c}(u)\left(G_{2}\right)$. Since $E^{c}\left(u^{\prime}\right)$ satisfies E5, $\left[\phi_{X}\right]^{c} \cap$ $\left[\phi_{Y}\right]^{c} \in E^{c}\left(u^{\prime}\right)\left(G_{1} \cup G_{2}\right)$. By the same argument as in the $G_{1} \cup G_{2} \neq N$ case, that means that $\left[\bigvee_{[t] \in X \cap Y} \phi_{[t]}\right]^{c} \in E^{c}\left(u^{\prime}\right)\left(G_{1} \cup G_{2}\right)$, and since $G_{1} \cup G_{2}=N$ that means that $X \cap Y \in$ $E^{f}([s])\left(G_{1} \cup G_{2}\right)$.

$G_{2}=N, G_{1}=\emptyset:$ Symmetric to the argument above.

$G_{1} \neq \emptyset, G_{2} \neq \emptyset, G_{1} \cup G_{2}=N$ : We have that for all $u$ such that $[u]=[s],\left[\phi_{X}\right]^{c} \in E^{c}(u)\left(G_{1}\right)$ and $\left[\phi_{Y}\right]^{c} \in$ $E^{c}(u)\left(G_{2}\right)$. Take $u=s$. Since $E^{c}(s)$ satisfies E5, $\left[\phi_{X}\right]^{c} \cap\left[\phi_{Y}\right]^{c} \in E^{c}(s)\left(G_{1} \cup G_{2}\right)$. By the same argument as above, $\left[\bigvee_{[t] \in X \cap Y} \phi_{[t]}\right]^{c} \in E^{c}(s)\left(G_{1} \cup G_{2}\right)$, and since $G_{1} \cup G_{2}=N$ that means that $X \cap Y \in E^{f}([s])\left(G_{1} \cup G_{2}\right)$.

Since $\phi$ is $C L C$-consistent, it will belong to at least one $s$ in $M^{c}$, hence to one of $[s]$ in $N^{f}$, hence by the truth lemma it is satisfied in $M^{f}$.

This concludes the proof of Theorem 1 .

Corollary 1 (Completeness of $C L C$ ) For any $\mathcal{C L C}$-formula $\phi, \models \phi$ implies $\vdash_{C L C} \phi$.

By ignoring the cases which involve $C_{G}$ operators in the completeness proof for $C L C$, we also get the following.

Corollary 2 (Completeness of $C L K)$ For any $\mathcal{C} \mathcal{L} \mathcal{K}$-formula $\phi$, $=\phi$ implies $\vdash_{C L K} \phi$.

\section{Epistemic Coalition Logic with Distributed Knowledge}

In this section we consider the logic $\mathcal{C} \mathcal{L} D$, extending coalition logic with individual knowledge operators and distributed knowledge.

The axiomatisation $C L D$ is obtained by extending $C L$ with the following standard axioms and rules for individual and distributed knowledge (see, e.g., [8]):

$\mathbf{K} K_{i}(\phi \rightarrow \psi) \rightarrow\left(K_{i} \phi \rightarrow K_{i} \psi\right)$

T $K_{i} \phi \rightarrow \phi$

$4 K_{i} \phi \rightarrow K_{i} K_{i} \phi$ 
$\mathbf{5} \neg K_{i} \phi \rightarrow K_{i} \neg K_{i} \phi$

$\mathbf{R N} \vdash_{C L D} \phi \Rightarrow \vdash_{C L D} K_{i} \phi$

DK $D_{G}(\phi \rightarrow \psi) \rightarrow\left(D_{G} \phi \rightarrow D_{G} \psi\right)$

DT $D_{G} \phi \rightarrow \phi$

D4 $D_{G} \phi \rightarrow D_{G} D_{G} \phi$

D5 $\neg D_{G} \phi \rightarrow D_{G} \neg D_{G} \phi$

D1 $K_{i} \phi \leftrightarrow D_{i} \phi$

D2 $D_{G} \phi \rightarrow D_{H} \phi$, if $G \subseteq H$

Again, soundness is straightforward.

Lemma 3 (Soundness) For any $\mathcal{C} \mathcal{L}$-formula $\phi, \vdash_{C L D} \phi$ implies $\models \phi$.

\subsection{Completeness}

In the remainder of this section we show that $C L D$ also is complete. An outline of the proof is as follows. As in the case of $C L C$, we start with the canonical model construction. However, rather than constructing a playable model, we construct a playable pseudomodel $M^{c}$. The truth lemma for the combined epistemic-coalitional language holds for $M^{c}$, but the relations interpreting distributed knowledge are not necessarily the intersections of the individual epistemic accessibility relations. The idea is to transform $M^{c}$ into a proper model, which has the E1-E6 properties, without breaking the truth lemma. This is done in two additional steps. First, $M^{c}$ is transformed into a finite pseudomodel $M^{f}$, as in the case of $C L C$. The transformation preserves satisfaction, as well as the playability properties (and E6 follows from finiteness). Using pseudomodels that are then transformed into proper models is a common way to deal with intersection in general and distributed knowledge in particular [31]. We can in fact now make directly use of an existing completeness result and proof for epistemic logic with distributed knowledge [8], by taking the (finite) epistemic pseudomodel underlying $M^{f}$ and transforming it into a proper (not necessarily finite) epistemic model which is used as the underlying epistemic model of the final model $M^{\prime}$. It remains to be shown that the transformation did not break the true playability properties, nor satisfaction of formulae in the closure.

Now, to the details.

For a set of formulae $s$, let $K_{a} s=\left\{K_{a} \phi: K_{a} \phi \in s\right\}$ and $D_{G} s=\left\{D_{G} \phi: D_{G} \phi \in s\right\}$.

Definition 1 (Canonical Playable Pseudomodel) The canonical playable pseudomodel $M^{c}=\left(S^{c},\left\{\sim_{i}^{c}: i \in\right.\right.$ $\left.N\},\left\{R_{G}^{c}: \emptyset \neq G \subseteq N\right\}, E^{c}, V^{c}\right)$ for $\mathcal{C} \mathcal{L D}$ is defined as follows:

- $S^{c}$ is the set of maximal $C L D$-consistent sets.

- $s \sim_{i}^{c}$ t iff $K_{i} s=K_{i} t$

- $s R_{G}$ iff $D_{H} s=D_{H}$ tfor all $H \subseteq G$

- $X \in E^{c}(s)(G)($ for $G \neq N)$ iff there exists $\psi$ such that $\left\{t \in S^{c}: \psi \in t\right\} \subseteq X$ and $[G] \psi \in s$.

- $X \in E^{c}(s)(N)$ iff $S^{c} \backslash X \notin E^{c}(s)(\emptyset)$.

- $V^{c}(s)=\{p: p \in s\}$

Lemma 4 (Pseudo Truth Lemma) For any formula $\phi, M^{c}, s=\phi$ iff $\phi \in s$.

Proof The proof is by induction on $\phi$. The epistemic cases are exactly as for standard normal modal logic. The case for coalition operators is exactly as in [25]. 
It is easy to check that $\sim_{i}^{c}$ are equivalence relations and E1-E5 hold for $E^{c}$.

Lemma 5 (Finite Pseudomodel) Every CLD-consistent formula $\phi$ is satisfied in a finite pseudomodel.

Proof The proof is exactly as in Theorem 1, namely the construction of $M^{f}$, but starting with a Canonical Playable Pseudomodel rather than Canonical Playable Model; the definition of $M^{c}$ contains the clause

$$
\Gamma R_{G} \Delta \text { iff } \forall H \subseteq G\left\{\psi: D_{H} \psi \in \Gamma\right\}=\left\{\psi: D_{H} \psi \in \Delta\right\}
$$

We add the following condition to the closure: $D_{i} \psi \in \operatorname{cl}(\phi)$ iff $K_{i} \psi \in \operatorname{cl}(\phi)$.

We define $M^{f}$ to be a pseudomodel instead of a model, by adding the clause:

$$
[s] R_{G}^{f}\left[s^{\prime}\right] \text { iff } \forall H \subseteq G\left\{\psi: D_{H} \psi \in[s]\right\}=\left\{\psi: D_{H} \psi \in\left[s^{\prime}\right]\right\}
$$

We show that $M^{f}$ is indeed a pseudomodel:

- $R_{i}^{f}=\sim_{i}^{f}$ : this follows from the fact that $K_{i} \phi \in[s]$ iff $D_{i} \phi \in[s]$ for any $i, \phi$ and $s$, which holds because of the $K_{i} \phi \rightarrow D_{i} \phi$ axiom and the new closure condition above.

- $G \subseteq H \Rightarrow R_{H}^{f} \subseteq R_{G}^{f}$ : this holds by definition.

We add a case for $\theta=D_{G} \psi$ to the inductive proof. This case is proven in exactly the same way as the $\theta=K_{i} \psi$ case: the definitions of $\sim_{i}^{f}$ and $R_{G}^{f}$ are of exactly the same form (in particular, $R_{G}^{f}$ is also an $S 5$ modality). The proof that E1-E6 hold in the resulting pseudomodel is the same as in the proof of Theorem 1 for $E^{f}$.

We are now going to transform the pseudomodel into a proper model; it is a well-known technique for dealing with distributed knowledge. In fact, we can make direct use of a corresponding existing result for epistemic logic with distributed knowledge, and extend it with the coalition operators/effectivity functions. We here give the more general result for the language with also common knowledge, which will be useful later.

Theorem 2 ([8]) If $M_{p}=\left(S,\left\{\sim_{i}: i \in N\right\},\left\{R_{G}: \emptyset \neq G \subseteq N\right\}, V\right)$ is an epistemic pseudomodel, then there is an epistemic model $M_{p}^{\prime}=\left(S^{\prime},\left\{\sim_{i}^{\prime}: i \in N\right\}, V^{\prime}\right)$ and a surjective (onto) function $\mathbf{f}: S^{\prime} \rightarrow S$ such that for every $s^{\prime} \in S^{\prime}$ and formula $\phi \in \mathcal{E} \mathcal{L C D}, M_{p}, \mathbf{f}\left(s^{\prime}\right) \models \phi$ iff $M_{p}^{\prime}, s^{\prime} \models \phi$.

Proof This result is directly obtained from the completeness proof for $\mathcal{E} \mathcal{L C D}$ sketched in [8, p. 70]. For a more detailed proof (for a more general language), see [31, Theorem 9].

Theorem 3 If a formula is satisfied in some finite pseudomodel, then it is satisfied in some model.

Proof Let $M=\left(S,\left\{\sim_{i}: i \in N\right\},\left\{R_{G}: \emptyset \neq G \subseteq N\right\}, E, V\right)$ be a finite pseudomodel such that $M, s \models \phi$. Let $M_{p}=\left(S,\left\{\sim_{i}: i \in N\right\},\left\{R_{G}: \emptyset \neq G \subseteq N\right\}, V\right)$ be the epistemic pseudomodel underlying $M$, and let $M_{p}^{\prime}=\left(S^{\prime},\left\{\sim_{i}^{\prime}: i \in N\right\}, V^{\prime}\right)$ and $\mathbf{f}: S^{\prime} \rightarrow S$ be as in Theorem 2. Let $\mathbf{f}^{-1}(X)=\left\{s^{\prime} \in S^{\prime}: \mathbf{f}\left(s^{\prime}\right) \in X\right\}$ for any set $X \subseteq S$. Finally, let $M^{\prime}=\left(S^{\prime},\left\{\sim_{i}^{\prime}: i \in N\right\}, E^{\prime}, V^{\prime}\right)$ where $E^{\prime}$ is defined as follows:

- For $G \neq N: Y \in E^{\prime}(u)(G) \Leftrightarrow \exists X \subseteq S,\left(Y \supseteq \mathbf{f}^{-1}(X)\right.$ and $\left.X \in E(\mathbf{f}(u))(G)\right)$

- for $G=N: Y \in E^{\prime}(u)(G) \Leftrightarrow \bar{Y} \notin E^{\prime}(u)(\emptyset)$

Two things must be shown: that $M^{\prime}$ is a proper model, and that it satisfies $\phi$.

Since $M_{p}^{\prime}$ is an epistemic model, to show that $M^{\prime}$ is a model all that remains to be shown is that $E^{\prime}$ is truly playable. We now show that that follows from true playability of $E$.

E1 Note that $\mathbf{f}^{-1}(X)=\emptyset$ iff $X=\emptyset$.

For $G \neq N, \emptyset \in E^{\prime}(u)(G)$ iff (by definition of $\left.E^{\prime}\right) \exists X \subseteq S,\left(\emptyset \supseteq \mathbf{f}^{-1}(X)\right.$ and $\left.X \in E(\mathbf{f}(u))(G)\right)$ iff $\emptyset \in E(\mathbf{f}(u))(G))$ which is impossible since $M$ satisfies E1. Note that in particular this proves $\emptyset \notin E^{\prime}(u)(\emptyset)$, which we will use in the E2 case below.

For $G=N, \emptyset \in E^{\prime}(u)(G)$ iff $S^{\prime} \notin E^{\prime}(u)(\emptyset)$ and we'll see that this is impossible in the E2 case below. 
E2 Note that $\mathbf{f}^{-1}(S)=S^{\prime}$.

For $G \neq N, S^{\prime} \in E^{\prime}(u)(G)$ iff (by definition of $\left.E^{\prime}\right) \exists X \subseteq S,\left(S^{\prime} \supseteq \mathbf{f}^{-1}(X)\right.$ and $X \in E(\mathbf{f}(u))(G)$ ), and by taking $X=S$ we get that $S^{\prime} \in E^{\prime}(u)(G)$ holds since $S^{\prime} \supseteq \mathbf{f}^{-1}(S)$ and $S \in E(\mathbf{f}(u))(G)$. Note that in particular this proves $S^{\prime} \in E^{\prime}(u)(\emptyset)$, which we needed in the $\mathrm{E} 1$ case above.

For $G=N, S^{\prime} \in E^{\prime}(u)(G)$ iff $\emptyset \notin E^{\prime}(u)(\emptyset)$ and this was proved in the E1 case above.

E3 $\forall u \in S^{\prime} \forall Y \subseteq S^{\prime} \bar{Y} \notin E^{\prime}(u)(\emptyset) \Rightarrow Y \in E^{\prime}(u)(N)$ follows immediately from the definition for $E^{\prime}(u)(N)$.

E4 $E^{\prime}$ is monotonic by definition for $G \neq N$.

For $N$, assume $X \subseteq Y$ and $X \in E^{\prime}(u)(N)$. Then $\bar{X} \notin E^{\prime}(u)(\emptyset)$. Since we already know that $E^{\prime}$ is monotonic for $G=\bar{\emptyset}$ and $\bar{Y} \subseteq \bar{X}, \bar{Y} \notin E^{\prime}(u)(\emptyset)$. So $Y \in E^{\prime}(u)(N)$.

E5 Let $u \in S^{\prime}, f(u)=s, G_{1}, G_{2} \subseteq N$ such that $G_{1} \cap G_{2}=\emptyset, X^{\prime}, Y^{\prime} \subseteq S^{\prime}, X^{\prime} \in E^{\prime}(u)\left(G_{1}\right)$ and $Y^{\prime} \in$ $E^{\prime}(u)\left(G_{2}\right)$. We must show that $X^{\prime} \cap Y^{\prime} \in E^{\prime}(u)\left(G_{1} \cup G_{2}\right)$. We reason by cases for $G_{1}$ and $G_{2}$.

First, consider the case that $G_{1} \cup G_{2} \neq N$. We must show that there is a $Z$ such that $\mathbf{f}^{-1}(Z) \subseteq X^{\prime} \cap Y^{\prime}$ and $Z \in E(s)\left(G_{1} \cup G_{2}\right)$. We have that there are $X, Y$ such that $\mathbf{f}^{-1}(X) \subseteq X^{\prime}$ and $X \in E(s)\left(G_{1}\right)$ and $\mathbf{f}^{-1}(Y) \subseteq Y^{\prime}$ and $Y \in E(s)\left(G_{2}\right)$. Take $Z=X \cap Y$. It is easy to see that $\mathbf{f}^{-1}(X \cap Y)=\mathbf{f}^{-1}(X) \cap \mathbf{f}^{-1}(Y)$ (from the defintion of $\mathbf{f}^{-1}(\cdot)$ ), and we thus get that $\mathbf{f}^{-1}(Z)=\mathbf{f}^{-1}(X) \cap \mathbf{f}^{-1}(Y) \subseteq X^{\prime} \cap Y^{\prime}$. From $X \in E(s)\left(G_{1}\right)$ and $Y \in E(s)\left(G_{2}\right)$ and superadditivity of $E$ we get that $Z \in E(s)\left(G_{1} \cup G_{2}\right)$.

Second, consider the case that $G_{1}=N$ or $G_{2}=N$. Without loss of generality, assume the former. That implies that $G_{2}=\emptyset$. We must show that $X^{\prime} \cap Y^{\prime} \in E^{\prime}(u)(N)$, i.e., that $\overline{X^{\prime} \cap Y^{\prime}} \notin E^{\prime}(u)(\emptyset)$. Assume otherwise, i.e., that $\overline{X^{\prime} \cap Y^{\prime}} \in E^{\prime}(u)(\emptyset)$, in other words that $\overline{X^{\prime}} \cup \overline{Y^{\prime}} \in E^{\prime}(u)(\emptyset)$. As $G_{2}=\emptyset$ we also have that $Y^{\prime} \in E^{\prime}(u)(\emptyset)$, and by E5 for $E^{\prime}$ for the case that $G_{1}=G_{2}=\emptyset \neq N$ (proven above) we get that $\left(\overline{X^{\prime}} \cup \overline{Y^{\prime}}\right) \cap Y^{\prime} \in E^{\prime}(u)(\emptyset)$. I.e., $\overline{X^{\prime}} \cap Y^{\prime} \in E^{\prime}(u)(\emptyset)$. By $\mathrm{E} 4$ for $E^{\prime}$ (proven above), we get that $\overline{X^{\prime}} \in E^{\prime}(u)(\emptyset)$. But that contradicts the fact that $X^{\prime} \in E(u)\left(G_{1}\right)$ with $G_{1}=N$.

Finally, consider the case that $G_{1} \cup G_{2}=N$ and $G_{1} \neq N$ and $G_{2} \neq N$. We must show that $X^{\prime} \cap Y^{\prime} \in$ $E^{\prime}(u)(N)$, i.e., that $\overline{X^{\prime} \cap Y^{\prime}} \notin E^{\prime}(u)(\emptyset)$, i.e., that there does not exist a $Z$ such that $\mathbf{f}^{-1}(Z) \subseteq \overline{X^{\prime} \cap Y^{\prime}}$ and $Z \in E(s)(\emptyset)$. Assume otherwise, that such a $Z$ exists. Let $X, Y$ be such that

$$
\begin{aligned}
& \mathbf{f}^{-1}(X) \subseteq X^{\prime} \text { and } X \in E(s)\left(G_{1}\right) \\
& \mathbf{f}^{-1}(Y) \subseteq Y^{\prime} \text { and } Y \in E(s)\left(G_{1}\right)
\end{aligned}
$$

which exist because $X^{\prime} \in E^{\prime}(u)\left(G_{1}\right)$ and $Y^{\prime} \in E^{\prime}(u)\left(G_{2}\right)$. From superadditivity of $E$ we get that

$$
X \cap Y \in E(s)(N)
$$

It follows that

$$
\overline{X \cap Y} \notin E(s)(\emptyset)
$$

because otherwise $\emptyset=(X \cap Y) \cap(\overline{X \cap Y}) \in E(s)(N)$ by E5 for $E$, which contradicts E1 for $E$. We furthermore have that

$$
\begin{aligned}
& \overline{X^{\prime}} \subseteq \overline{\mathbf{f}^{-1}(X)} \subseteq \mathbf{f}^{-1}(\bar{X}) \\
& \overline{Y^{\prime}} \subseteq \overline{\mathbf{f}^{-1}(Y)} \subseteq \mathbf{f}^{-1}(\bar{Y})
\end{aligned}
$$

which follow immediately from the facts that $\mathbf{f}^{-1}(X) \subseteq X^{\prime}$ and $\mathbf{f}^{-1}(Y) \subseteq Y^{\prime}$ and the definition of $\mathbf{f}^{-1}(\cdot)$. From (3) it follows that

$$
\overline{X^{\prime}} \cup \overline{Y^{\prime}} \subseteq \mathbf{f}^{-1}(\bar{X} \cup \bar{Y})
$$

From (4) and the assumption that $Z \in E(s)(\emptyset)$ we get that $\mathbf{f}^{-1}(Z) \subseteq \mathbf{f}^{-1}(\bar{X} \cup \bar{Y})$, and it follows, by surjectivity of $\mathbf{f}$, that

$$
Z \subseteq \overline{X \cap Y}
$$

By (5) and the assumption that $Z \in E(s)(\emptyset)$ we get that $\overline{X \cap Y} \in E(s)(\emptyset)$. But this contradicts (2). 
E6 We must show that $E^{\prime n c}(u)(\emptyset) \neq \emptyset$, for any $u$. Let $s=f(u)$, and let $X \in E^{n c}(s)(\emptyset)$ (exists because of E6 for $E$ ). We show that $\mathbf{f}^{-1}(X) \in E^{\prime} n c(u)(\emptyset)$. First, we have that $\mathbf{f}^{-1}(X) \in E^{\prime}(u)(\emptyset)$; this follows from the fact that $X \in E(s)(\emptyset)$ and the definition of $E^{\prime}$. Second, assume, towards a contradiction, that there exists a $Y \subsetneq \mathbf{f}^{-1}(X)$ such that $Y \in E^{\prime}(u)(\emptyset)$. By the definition of $E^{\prime}$, this means that there is a $Z$ such that $\mathbf{f}^{-1}(Z) \subseteq Y$ and $Z \in E(s)(\emptyset)$. Since $Y \subsetneq \mathbf{f}^{-1}(X)$ and $\mathbf{f}^{-1}(Z) \subseteq Y$ it follows that $\mathbf{f}^{-1}(Z) \subsetneq \mathbf{f}^{-1}(X)$. It is easy to see (from surjectivity of $\mathbf{f}$ ) that it follows that $Z \subsetneq X$, and this contradicts the assumption that $Z \in E(s)(\emptyset)$ and $X \in E^{n c}(s)(\emptyset)$.

In order to show that $M^{\prime}$ satisfies $\phi$, we show that $M, \mathbf{f}(u) \models \gamma$ iff $M^{\prime}, u \models \gamma$ for any $u \in S^{\prime}$ and any $\gamma$, by induction in $\gamma$. All cases except $\gamma=[G] \psi$ are exactly as in the proof of Theorem 2.

For the case that $\gamma=[G] \psi$, the inductive hypothesis is that for all proper subformulae $\chi$ of $[G] \psi$, and any $v$, $M, \mathbf{f}(v) \models \chi$ iff $M^{\prime}, v \models \chi$. We can state this as $\left\{v: M^{\prime}, v \models \chi\right\}=\mathbf{f}^{-1}\left(\chi^{M}\right)$, or $\chi^{M^{\prime}}=\mathbf{f}^{-1}\left(\chi^{M}\right)$.

First, consider the case that $G \neq N$. Let $\mathbf{f}(u)=s . M^{\prime}, u=[G] \psi$ iff $\psi^{M^{\prime}} \in E^{\prime}(u)(G)$ iff there is an $X$ such that $\mathbf{f}^{-1}(X) \subseteq \psi^{M^{\prime}}$ and $X \in E(s)(G)$. This holds iff $\psi^{M} \in E(s)(G)$ iff $M, s=[G] \psi$. For the implication to the left take $X=\psi^{M}$; for the implication to the right observe that $\mathbf{f}^{-1}(X) \subseteq \mathbf{f}^{-1}\left(\psi^{M}\right)$ implies that $X \subseteq \psi^{M}$, and $\psi^{M} \in E(s)(G)$ follows from $X \in E(s)(G)$ by outcome monotonicity of $E$.

Second, consider the case that $G=N . M, s=[N] \psi$ iff $\psi^{M} \in E(s)(N)$ iff (*) $\neg \psi^{M} \notin E(s)(\emptyset)$ iff (as above) $\neg \psi^{M^{\prime}} \notin E^{\prime}(u)(\emptyset)$ iff $M^{\prime}, u \models[N] \psi$. (*): one direction E3, the other direction E5 and E1.

Corollary 3 (Completeness of $C L D$ ) For any $\mathcal{C L D}$-formula $\phi$, $\models \phi$ implies $\vdash_{C L D} \phi$.

\section{Epistemic Coalition Logic with both Common and Distributed Knowl- edge}

In this section we consider the logic $\mathcal{C} \mathcal{L C D}$, extending coalition logic with operators for individual knowledge, common knowledge and distributed knowledge.

The axiomatisation $C L C D$ is obtained by extending $C L$ with the axioms and rules of $C L C$ and $C L D$.

Lemma 6 (Soundness) For any $\mathcal{C} \mathcal{L C D}$-formula $\phi, \vdash_{C L C D} \phi$ implies $\mid=\phi$.

Completeness can in fact be shown in exactly the same way as for $C L D$, except that there is an extra clause for $C_{G} \phi$ in the proof of satisfaction which is taken care of in the same way as in the proof for $C L C$.

Theorem 4 Any CLCD-consistent formula is satisfied in some finite pseudomodel.

Proof The proof is identical to the proof of Lemma 5, starting with the canonical playable pseudomodel, with the addition of the inductive clause $\theta=C_{G} \psi$ as in the proof of Theorem 1 .

We can now use the same approach as in the case of $\mathcal{C} \mathcal{L D}$.

Theorem 5 If a $\mathcal{C} \mathcal{L C D}$ formula is satisfied in some finite pseudomodel, it is satisfied in some model.

Proof The proof goes exactly like the proof of Theorem 3, using Theorem 2. The definition of the model $M^{\prime}$ is identical to the definition in Theorem 3, as is the proof that it is a proper model. For the last part of the proof, i.e., showing that $M^{\prime}$ satisfies $\phi$, note that the last clause in Theorem 2 holds for epistemic logic with both distributed and common knowledge. Thus, the proof is completed by only adding the inductive clause for $[G] \phi$, which is done in exactly the same way as in Theorem 3. 
Corollary 4 (Completeness of $C L C D$ ) For any $\mathcal{C L C D}$-formula $\phi$, $\models \phi$ implies $\vdash_{C L C D} \phi$.

\section{Computational Complexity}

The following complexity result is an easy consequence of known results for other logics:

Theorem 6 The satisfiability problem for $\mathcal{C} \mathcal{L C}$ and for $\mathcal{C L C D}$ is EXPTIME-complete.

Proof EXPTIME-hardness follows from EXPTIME-hardness of $\mathcal{S} 5_{n}+C$ [16]. EXPTIME upper bound follows from the upper bound for $\mathcal{A} \mathcal{T} \mathcal{E} \mathcal{L}$ [30].

The next result is much less obvious.

Theorem 7 The satisfiability problem for $\mathcal{C} \mathcal{L} D$ is PSPACE-complete.

Proof PSPACE-hardness follows from PSPACE-hardness of $\mathcal{S} 5_{n}$ [16] and also from PSPACE-hardness of $\mathcal{C} \mathcal{L}$ $[25]$.

We now consider the PSPACE upper bound. We first define a notion of a tableau for a $\mathcal{C} \mathcal{L} \mathcal{D}$ formula. We show that a $\mathcal{C L D}$ formula $\phi$ is satisfiable if, and only, if, there is a $\mathcal{C} \mathcal{L D}$ tableau for it (Lemma 7). Then we give a procedure $H M P$ that given a $\mathcal{C} \mathcal{L}$ formula $\phi$, attempts to construct a $\mathcal{C} \mathcal{L}$ tableau for it. We prove that this construction succeeds if and only if $\phi$ is satisfiable (Lemma 9). The structure constructed by $H M P(\phi)$ is exponential in $|\phi|$, however we show that there is an algorithm that checks whether $\operatorname{HMP}(\phi)$ will return true that runs in space polynomial in $|\phi|$.

Before defining a $\mathcal{C L D}$ tableau we need a notion of a closure of a $\mathcal{C} \mathcal{L}$ formula $\phi$, to be used in the tableau construction. Without loss of generality, we assume that in $\phi$ all modalities $K_{i}$ are replaced with $D_{i}$ and the only propositional connectives in $\phi$ are $\neg$ and $\wedge$. Let $\operatorname{ccl}(\phi)$ be the smallest set of formulas satisfying the following conditions:

ccl1 $\operatorname{ccl}(\phi)$ contains all subformulas of $\phi$

ccl2 $\operatorname{ccl}(\phi)$ is closed under single negations

$\mathbf{c c l 3}[N] \psi \in \operatorname{ccl}(\phi) \Rightarrow[\emptyset] \neg \psi \in \operatorname{ccl}(\phi)$

ccl4 $[\emptyset] \psi \in \operatorname{ccl}(\phi) \Rightarrow[N] \neg \psi \in \operatorname{ccl}(\phi)$

Note that the size (the number of formulas) of $\operatorname{ccl}(\phi)$ is at most $2|\phi|$, hence it is polynomial in $|\phi|$ (unlike the closure for $\mathcal{C} \mathcal{L}$ formulas defined in [25]; the latter was first observed to be exponential by Hoang Nga Nguyen and corrected in [24], a published version is in [3]).

Definition $2 A \mathcal{C L D}$ tableau for a formula $\phi$ is a tuple $T=\left(S, L, R_{1}, \ldots, R_{n}\right)$, where $S$ is a set of states, $R_{i}$ for each agent $i$ is a binary relation on $S$, and $L$ is a labelling function which associates with each state $s \in S$ a set $L(s) \subseteq \operatorname{ccl}(\phi)$ of formulas such that

T0 For some state $s_{0} \in S, \phi \in L\left(s_{0}\right)$

PT $L(s)$ is a fully expanded propositional tableau, that is, a set of formulas satisfying

PT(a) if $\neg \neg \psi \in L(s)$ then $\psi \in L(s)$;

$\mathbf{P T}(\mathbf{b})$ if $\psi \wedge \psi^{\prime} \in L(s)$, then $\psi, \psi^{\prime} \in L(s)$;

PT(c) if $\neg\left(\psi \wedge \psi^{\prime}\right) \in L(s)$, then either $\neg \psi \in L(s)$ or $\neg \psi^{\prime} \in L(s)$; and

PT(d) $L(s)$ does not contain $\psi$ and $\neg \psi$ for any $\psi$

PT(e) for every $\psi \in L(s)$, and every subformula $\psi^{\prime}$ of $\psi$, either $\psi^{\prime} \in L(s)$ or $\neg \psi^{\prime} \in L(s)$

ET1 if $D_{G} \psi \in L(s)$, then $\psi \in L(s)$ 
ET2 if $\neg D_{G} \psi \in L(s)$, then there exists $t$ with $(s, t) \in R_{i}$ for all $i \in G$ and $\neg \psi \in L(t)$

ET3 if $(s, t) \in R_{i}$ for every $i \in G$, then $D_{H} \psi \in L(s)$ iff $D_{H} \psi \in L(t)$ for every $H \subseteq G$

CT1 If $[N] \psi \in L(s)$, then $\neg[\emptyset] \neg \psi \in L(s)$, and if $\neg[\emptyset] \neg \psi \in L(s)$, then $[N] \psi \in L(s)$

CT2 if $\left[G_{1}\right] \psi_{1}, \ldots,\left[G_{k}\right] \psi_{k} \in L(s)$ for $k>0$ where $G_{i}$ are pairwise disjoint, then there exists $s^{\prime} \in S$ such that $\left\{\psi_{1}, \ldots, \psi_{k}\right\} \subseteq L\left(s^{\prime}\right)$

CT3 if $\left[G_{1}\right] \psi_{1}, \ldots,\left[G_{k}\right] \psi_{k}, \neg[G] \psi \in L(s)$, where $G_{i}$ are pairwise disjoint and non-empty, $\bigcup_{i} G_{i} \subseteq G$, then there exists $s^{\prime} \in S$ such that $\left\{\psi_{1}, \ldots, \psi_{k}, \neg \psi\right\} \subseteq L\left(s^{\prime}\right)$

Intuitively, ET1 - ET3 describe epistemic conditions on a tableau, and CT1 - CT3 conditions ensuring satisfiability in coalition logic and closely correspond to conditions on a function $v$ used by Pauly to define a satisfiabilitychecking game for $\mathcal{C} \mathcal{L}$ in [25].

The structure that we refer to as a tableau following [16] is also called a Hintikka set or Hintikka structure (see for example [2]).

Observe that since $\operatorname{ccl}(\phi)$ contains finitely many formulas, a $\mathcal{C} \mathcal{L} \mathcal{D}$ tableau for $\phi$ can always be assumed to be finite (intuitively, if there is an infinite tableau for $\phi$, then it can be transformed into a finite tableau by identifying all the states with the same label $L(s)$, since there are finitely many possible $L(s) \subseteq \operatorname{ccl}(\phi)$. It is easy to check that the resulting structure still satisfies all the conditions of Definition 2.)

Lemma $7 A \mathcal{C L D}$ formula $\phi$ is satisfiable if, and only if, there is a $\mathcal{C} \mathcal{L} \mathcal{D}$ tableau $T=\left(S, L, R_{1}, \ldots, R_{n}\right)$ for $\phi$.

Proof The direction from left to right (if a formula has a model, then it has a tableau) is relatively routine and is left out. It follows the proof in [16] for the epistemic part and in [25] and [24] for the coalition logic part.

The difficult direction is constructing a model given a tableau. Suppose $T=\left(S, L, R_{1}, \ldots, R_{n}\right)$ is a tableau for $\phi$, in particular $\phi \in s_{0}$ for some $s_{0} \in S$. We construct $M=\left(S, E,\left\{\sim_{i}: i \in N\right\}, V\right)$ as follows. The set of states $S$ is the same as in $T$. For each $i \in N, \sim_{i}$ is the reflexive, symmetric, transitive closure of $R_{i} . V(s)=L(s) \cap \Theta^{4}$. For convenience, we introduce an abbreviation $J(\psi)=\{s: \psi \in L(s)\}$.

Finally, we define $E$ as follows: $X \in E(s)(G)$ for $G \neq N$ iff $X=S$ or there exist $\left[G_{1}\right] \psi_{1}, \ldots,\left[G_{k}\right] \psi_{k} \in$ $\operatorname{ccl}(\phi), k>0$, such that all of the following properties hold:

(def1) $G_{1} \cup \ldots \cup G_{k} \subseteq G$

(def2) $G_{i}$ are pairwise disjoint

(def3) $\bigcap_{i} J\left(\psi_{i}\right) \subseteq X$

(def4) $\left[G_{1}\right] \psi_{1}, \ldots,\left[G_{k}\right] \psi_{k} \in L(s)$

$X \in E(s)(N)$ iff $\bar{X} \notin E(s)(\emptyset)$.

We need to prove that $E$ so defined satisfies conditions E1-E6.

E1 E1 holds for $G \neq N$ and $s \in S$ because if for some $\psi_{1}, \ldots, \psi_{k}$ such that they conform to the definition of $E$, we had $\bigcap_{i} J\left(\psi_{i}\right) \subseteq \emptyset$ (by def3) then by CT2, $\left\{\psi_{1}, \ldots, \psi_{k}\right\}$ would have to belong to a label of some state $s^{\prime} \in S$ hence $\bigcap_{i} J\left(\psi_{i}\right) \neq \emptyset$.

E1 for $G=N: \emptyset \notin E(s)(N)$ because by construction $S \in E(s)(\emptyset)$.

E2 E2 holds for $G \neq N$ by construction. Since by E1 for $\emptyset, \emptyset \notin E(s)(\emptyset)$, by construction of $E$ for $N, S \in$ $E(s)(N)$.

E3 E3 holds in all states by construction of $E$ for $N$.

\footnotetext{
${ }^{4}$ Recall that $\Theta$ is the set of propositional variables.
} 
E4 E4 holds for $G \neq N$ because due to (def3) if $X \in E(s)(G)$, then $Y \in E(s)(G)$ for any $Y \supseteq X)$ (in other words, $E$ is monotonic by construction). For the case of $N$, let $X \in E(s)(N)$ and $X \subseteq Y$. By construction, $\bar{X} \notin E(s)(\emptyset)$. Suppose by contradiction that $\bar{Y} \in E(s)(\emptyset)$. Since $\bar{Y} \subseteq \bar{X}$ and $E(s)(\emptyset)$ is monotonic due to (def3), $\bar{X} \notin E(s)(\emptyset)$ : a contradiction. Hence $\bar{Y} \notin E(s)(\emptyset)$ and $Y \in E(s)(N)$.

E5 Let $G \cap H=\emptyset$ and $X \in E(s)(G)$ and $Y \in E(s)(H)$. Suppose $G$ and $H$ are non-empty and $X, Y$ are different from $S$. By the construction of $E$, there are $G_{1}, \ldots, G_{k}$ and $H_{1}, \ldots, H_{m}$ such that $\left[G_{i}\right] \psi_{i}$ and $\left[H_{j}\right] \chi_{j}$ conform to (def1)-(def4). Note also that since $G$ and $H$ are disjoint and $G_{i} \subseteq G$ and $H_{j} \subseteq H, G_{i}$ and $H_{j}$ are also all pairwise disjoint. It is an easy consequence of (def3) for both $\psi_{i}$ and $\chi_{j}$ that $\bigcap_{i} J\left(\psi_{i}\right) \cap \bigcap_{j} J\left(\chi_{j}\right) \subseteq$ $X \cap Y$ and hence $X \cap Y \subseteq E(s)(G \cup H)$. If $Y=S, X \cap Y=X$ and we can use $G \subseteq G \cup H$ to show that $X \cap Y \subseteq E(s)(G \cup H)$ holds (just replace $\bigcup_{i} G_{i} \subseteq G$ with $\bigcup_{i} G_{i} \subseteq G \cup H$ ). For $G$ and $H$ both empty, the argument is similar to above.

For $G=\emptyset$ and $H=N$, since $G \cup H=N$ so we need to show $X \cap Y \in E(s)(N)$. Assume to the contrary that $\overline{X \cap Y} \in E(s)(\emptyset)$. Then $X \cap \bar{Y} \in E(s)(\emptyset)$ by superadditivity of $E(s)(\emptyset)$ shown above, and $\bar{Y} \in E(s)(\emptyset)$ by monotonicity of $E(s)(\emptyset)$, so $Y \notin E(s)(N)$, a contradiction.

E6 E6 holds because $M$ is finite.

Now we prove that for every $\psi \in \operatorname{ccl}(\phi), \psi \in L(s)$ implies $M, s \models \psi$ and $\neg \psi \in L(s)$ implies $M, s \mid \neg \psi$. The proof proceeds by induction on the length of $\psi$.

case $\psi=p$ immediate since $p \in V(s)$ by construction iff $p \in L(s)$.

case booleans follows easily by induction since $L(s)$ is a fully expanded propositional tableau.

case $\psi=D_{G} \chi$ Suppose $\neg D_{G} \chi \in L(s)$. Then by ET2, there exists $t \in S$ such that $R_{i}(s, t)$ holds for all $i \in G$ and $\neg \chi \in L(t)$. Since for every $i, R_{i} \subseteq \sim_{i}, s \sim_{i} t$ holds for all $i \in G$, hence $s$ and $t$ are connected by $\bigcap_{i} \sim_{i}$. By the inductive hypothesis, $M, t \models \neg \chi$, so $M, s \models \neg D_{G} \chi$.

Let $D_{G} \chi \in L(s)$. We need to show that for all $t$ such that $s \sim_{i} t$ for all $i \in G, \chi \in L(t)$ (this will give $M, t=\chi$ by the inductive hypothesis, and hence $\left.M, s \models D_{G} \chi\right)$. In a simple case when $R_{i}(s, t)$ holds for all $i \in G$, we know that $D_{G} \chi \in L(t)$ by ET3, and then by ET1, $\chi \in L(t)$. Now suppose that $R_{i}(s, t)$ for some or all $i \in G$ does not hold, but $(s, t)$ belongs to a reflexive symmetric transitive closure of $R_{i}$ for all $i \in G$. If $s \sim_{i} t$ for each $i \in G$ is added by reflexivity, that is, $s=t$, then $\chi \in L(t)$ by ET1. Otherwise, there is a chain $s_{0}, s_{1}, \ldots, s_{k}$ in $T$ such that $s_{0}=s, s_{k}=t$, and for every pair $s_{j}, s_{j+1}$, either $R_{i}\left(s_{j}, s_{j+1}\right)$ holds or $R_{i}\left(s_{j+1}, s_{j}\right)$, for all $i \in G$. In either case, $D_{G} \chi \in L\left(s_{j}\right)$ iff $D_{G} \chi \in L\left(s_{j+1}\right)$, hence $\chi \in L\left(s_{j}\right)$ iff $\chi \in L\left(s_{j+1}\right)$, hence by induction $\chi \in L(t)$, so $M, t \models \chi$ by the inductive hypothesis, and hence $M, s \models D_{G} \chi$.

case $\psi=[G] \chi$

We need to show that for any $[G] \chi \in \operatorname{ccl}(\phi)$, if $[G] \chi \in L(s)$, then $J(\chi) \in E(s)(G)$, and if $J(\chi) \in E(s)(G)$, then $[G] \chi \in L(s)$.

First we prove both directions for $G \neq N$. Let $[G] \chi \in L(s)$, then $J(\chi) \in E(s)(G)$ by construction of $E$.

Suppose $J(\chi) \in E(s)(G)$. If $J(\chi)=S$, suppose by contradiction that $\neg[G] \chi \in L(s)$, then by CT3 $\neg \chi$ should be in $L\left(s^{\prime}\right)$ for some $s^{\prime} \in S$ : a contradiction with $J(\chi)=S$.

If $J(\chi) \neq S$, then there are $\left[G_{1}\right] \psi_{1}, \ldots,\left[G_{k}\right] \psi_{k}$ satisfying conditions (def1)-(def4), in particular $\bigcap_{i} J\left(\psi_{i}\right) \subseteq$ $J(\chi)$ and $\left[G_{1}\right] \psi_{1}, \ldots,\left[G_{k}\right] \psi_{k} \in L(s)$. Assume by contradiction that $\neg[G] \chi \in L(s)$. Since $\left[G_{1}\right] \psi_{1}, \ldots,\left[G_{k}\right] \psi_{k}, \neg[G] \chi \in$ $L(s)$, by CT3, $\left\{\psi_{1}, \ldots, \psi_{k}, \neg \psi\right\} \subseteq L\left(s^{\prime}\right)$ for some $s^{\prime} \in S$. This means that there is $s^{\prime} \in \bigcap_{i} J\left(\psi_{i}\right)$ such that $s^{\prime} \notin J(\chi)$ : a contradiction with $\bigcap_{i} J\left(\psi_{i}\right) \nsubseteq J(\chi)$. So $[G] \chi \in L(s)$.

Now let us consider $G=N$. If $[N] \chi \in L(s)$, then by CT1, $\neg[\emptyset] \neg \chi \in L(s)$. By the proof above for $G \neq N$, $J(\neg \chi) \notin E(s)(\emptyset)$. This implies $\overline{J(\chi)} \notin E(s)(\emptyset)$, hence $J(\chi) \in E(s)(N)$.

If $J(\chi) \in E(s)(N)$, then by construction $J(\neg \chi) \notin E(s)(\emptyset)$. By the preceding argument, $[\emptyset] \neg \chi \notin L(s)$. By $\mathrm{CT} 1,[N] \chi \in L(s)$. 
Next we define a procedure $H M P$ for constructing a tableau for a $\mathcal{C L D}$ formula $\phi$. The terminology is mainly taken from [16]. Since [16] gives a procedure for $\mathcal{S} 5_{n}$ and only sketch modifications required for adding $D$ for the grand coalition, we adapted a rule for $D_{G}$ from the tableaux algorithm for multi-agent epistemic logics with $C_{G}$ an $D_{G}$ modalities for arbitrary coalitions proposed in [2].

We need the following terminology to define $H M P . \psi \in \Gamma$ is a witness that $\Gamma$ is not a propositional tableau if one of the clauses PT(a) - PT(c) with $\Gamma$ in place of $L(s)$ does not apply to $\psi$; similarly $\psi$ is a witness that $\Gamma$ is not fully expanded if PT(e) does not hold for $\psi ; \Gamma$ is blatantly inconsistent if $\mathrm{PT}(\mathrm{d})$ with $\Gamma$ in place of $L(s)$ is violated.

The procedure below constructs a pre-tableau, and we will show later (in the proof of Lemma 9) how to construct a tableau from the result. A pre-tableau is a graph with two kinds of nodes: if $L(s)$ is a fully expanded propositional tableau, we call $s$ a state, otherwise it is an internal node. There are three kinds of edges: $p t$ edges, $i$ edges for $i \in N$, and $c t$ edges. $p t$ edges and $c t$ edges form a tree, and $i$ edges 'almost' form a tree (two nodes can have multiple $i$ edges between them, and $i$ edges are intended to be symmetric and transitive, so a node can have a back edge to an $i$-ancestor). To be precise, $i$ edges form a symmetric transitive tree where edges are labelled by sets $G$ of agents.

\section{Procedure $H M P(\phi)$}

1. Construct a tree consisting of a single root node $s_{0}$ with $L\left(s_{0}\right)=\{\phi\}$.

2. Repeat each of (a)-(d) below exhaustively until none of (a)-(d) applies:

\section{(a) Creating pt-successor nodes:}

i. if $s$ is a leaf of the tree, $L(s)$ is not blatantly inconsistent, $L(s)$ is not a propositional tableau, and $\psi$ is the first (in some lexicographic ordering of formulas in $L(s)$ ) witness to this fact, then

A. if $\psi$ is of the form $\neg \neg \psi^{\prime}$, then create a $p t$ child $s^{\prime}$ of $s$ in the tree and set $L\left(s^{\prime}\right)=L(s) \cup\left\{\psi^{\prime}\right\}$

B. if $\psi$ is of the form $\psi_{1} \wedge \psi_{2}$, then create a pt child $s^{\prime}$ of $s$ in the tree and set $L\left(s^{\prime}\right)=L(s) \cup$ $\left\{\psi_{1}, \psi_{2}\right\}$

C. if $\psi$ is of the form $\neg\left(\psi_{1} \wedge \psi_{2}\right)$, then create two $p t$ children $s_{1}$ and $s_{2}$ of $s$ and set $L\left(s_{i}\right)=$ $L(s) \cup\left\{\neg \psi_{i}\right\}$.

ii. $s$ is a leaf of the tree, $L(s)$ is not blatantly inconsistent, $L(s)$ is not a fully expanded propositional tableau, and $\psi$ is the first (in some lexicographic ordering of subformulas of formulas in $L(s)$ ) witness to this fact, then create two pt-children $s_{1}$ and $s_{2}$ of $s$ and set $L\left(s_{1}\right)=L(s) \cup\{\psi\}$, $L\left(s_{2}\right)=L(s) \cup\{\neg \psi\}$.

(b) Creating $i$-successor nodes: $:^{5}$ if $s$ is a leaf of the tree and $L(s)$ is a fully expanded propositional tableau, for each $\psi$ such that $\neg D_{G} \psi \in L(s)$ let

$$
\begin{gathered}
L^{\prime \prime}(s, \psi)=\left\{D_{H} \psi^{\prime}: D_{H} \psi^{\prime} \in L(s) \text { and } H \subseteq G\right\} \cup \\
\left\{\neg D_{H} \psi^{\prime}: \neg D_{H} \psi^{\prime} \in L(s) \text { and } H \subseteq G\right\} \cup\{\neg \psi\}
\end{gathered}
$$

If there is no node $s^{\prime \prime}$ in the tree that is an $i$ ancestor of $s^{6}$ for all $i \in G$, or $s^{\prime \prime}=s$, such that $L^{\prime \prime}(s, \psi) \subseteq L\left(s^{\prime \prime}\right)$, then create a node $s^{\prime}$ with $L\left(s^{\prime}\right)=L^{\prime \prime}(s, \psi)$ and create an $i$-edge between $s$ and $s^{\prime}$ for all $i \in G$.

(c) Creating ct-successor nodes:

i. for each maximal subset of $L(s)$ of the form $\left[G_{1}\right] \psi_{1}, \ldots,\left[G_{k}\right] \psi_{k}$ where $G_{i}$ are pairwise disjoint, create a $c t$-successor $s^{\prime}$ with $L\left(s^{\prime}\right)=\left\{\psi_{1}, \ldots, \psi_{k}\right\}$

ii. for each maximal subset of $L(s)$ of the form $\neg[G] \psi,\left[G_{1}\right] \psi_{1}, \ldots,\left[G_{k}\right] \psi_{k}$ where $G_{i}$ are pairwise disjoint and non-empty and $\bigcup_{i} G_{i} \subseteq G$, create a $c t$-successor $s^{\prime}$ with $L\left(s^{\prime}\right)=\left\{\neg \psi, \psi_{1}, \ldots, \psi_{k}\right\}$

\footnotetext{
${ }^{5}$ This step creates a witness for each formula of the form $\neg D_{G} \psi$ in $L(s)$, that is a state $t$ epistemically connected to $s$ for all $i \in G$ and such that $\neg \psi \in L(t)$. This makes sure that condition ET2 of Definition 2 is satisfied. Note that if $s$ and $t$ are epistemically connected for all agents in $G$, then they are epistemically connected for all agents in $H \subseteq G$, so it should hold that $D_{H} \psi^{\prime} \in L(s)$ iff $D_{H} \psi^{\prime} \in L(t)$.

${ }^{6} \mathrm{By}$ an $i$-ancestor of a node $s$ we mean a node $t$ such that a path from $t$ to $s$ contains only $i$ edges and $p t$ edges.
} 
(d) Marking nodes satisfiable: if $s$ is not marked satisfiable then mark $s$ satisfiable if either

i. $L(s)$ is not a fully expanded propositional tableau and some $p t$ successor $s^{\prime}$ of $s$ is marked satisfiable, or $L(s)$ is a fully expanded propositional tableau and

A. $L(s)$ does not contain $D_{G} \psi$ and $\neg \psi$ for any $\psi$

B. $L(s)$ does not contain a pair of formulas $[\emptyset] \psi,[N] \neg \psi$ or a pair of formulas $[\emptyset] \neg \psi,[N] \psi \in$ $L(s)$, for any $\psi$

C. $L(s)$ is not blatantly inconsistent

D. either $s$ has no $i$-successors or $c t$-successors and no rules for creating $i$ or $c t$ successors are applicable

E. or $s$ has $i$-successors and/or $c t$-successors and all of them are marked satisfiable.

(e) If the root of the tree is marked satisfiable, then return true, otherwise return false.

\section{Lemma 8 The HMP procedure terminates.}

Proof To show termination, we will show that the depth of the tree costructed by $H M P$ is bounded by $O\left(|\phi|^{3}\right)$ and the branching factor is bounded by $O\left(3^{|\phi|}\right)$. Observe that $\operatorname{ccl}(\phi)$ has size (cardinality) at most $2|\phi|$, and that a consistent $L(s)$ can have size (cardinality) at most $|\phi|$.

Consider a branch of the pre-tableau. It consists of $p t, i$ and $c t$ edges. Intuitively, every time an $i$ or $c t$ edge is added, it leads from a state to an internal node. The internal node will have a path of $p t$ edges from it to another state, from where again an $i$ or a $c t$ edge will be added. The maximal length of such a $p t$ path between an internal node $s$ and a state $t$ (starting with a singleton set $L(s)$ and adding at most $|\phi|-1$ formulas to obtain a fully expanded propositional tableau $L(t))$ is $|\phi|$. The number of $c t$ edges on a path is bounded by the number of nestings of coalitional modalities in $\phi$, which is at most $|\phi|$ (since each $c t$ edge reduces the depth of nesting of coalitional modalities by 1). Now consider $i$ edges, or tuples of parallel $i$ edges for various $D_{G}, G \subseteq N$ added by step 2(b). Note that there are only at most $|\phi| \operatorname{such} D_{G}$ occurring in $\operatorname{ccl}(\phi)$. If $s$ and $t$ are connected by $\bigcap_{i \in G}$ $i$ edges, then the modal depth for $D_{H} \chi \in L(t)$ formulas, for $H \subseteq G$, in $\bigcap_{i \in H} i$-successors does not change. However the maximal number of consecutive $\bigcap_{i \in H} i$ successors on a path is $|\phi|$ (because there can be no more than $|\phi|$ distinct states $s, t$ on an $\bigcap_{i \in H^{-}}$path with $\left\{D_{H} \psi: D_{H} \psi \in L(s)\right\}=\left\{D_{H} \psi: D_{H} \psi \in L(t)\right\}$ ), and the modal depth of $L(s)$ decreases after an intervening $\bigcap_{i \in H} i$ step where $H \nsubseteq G$ step, or a $c t$-step. The maximal length of a path is thus $O\left(|\phi|^{3}\right)$.

The branching factor of the pre-tableau is possibly exponential because of the number of $c t$-children of a node. The number of maximal subsets of the form $\{G:[G] \psi \in L(s)\}$ such that all members of the subset are disjoint can be exponential in the number of formulas in $L(s), 3^{|L(s)| / 3}$ to be precise; the argument is as in [23] for the number of cliques in a graph (one can think of $G_{i}$ as vertices, and there is an edge between $G_{i}$ and $G_{j}$ if they are disjoint).

Since there is a bound on both the branching factor and the depth of the tree constructed by $H M P(\phi)$, the procedure terminates.

\section{Lemma $9 A \mathcal{C L D}$ formula $\phi$ is satisfiable if and only if $H M P(\phi)$ returns true.}

Proof First suppose that $H M P(\phi)$ returns true. We show how to construct a tableau for $\phi$ using the pre-tableau constructed by $H M P(\phi)$. A tableau is obtained by keeping only the states marked satisfiable and dropping all $p t$ and $c t$ edges. $R_{i}$ edges are added as follows. If $s$ and $t$ are states, and there is a path of $s, s_{1}, \ldots, s_{k}, t$ between them, where $s_{1}, \ldots, s_{k}$ are internal nodes, there is an $i$ edge between $s$ and $s_{1}$, and the rest of the edges are $p t$ edges, then add $R_{i}(s, t)$. Then take a reflexive symmetric transitive closure of each $R_{i}$. Observe that ET1 holds because only nodes marked satisfiable are retained, and ET2 and ET3 hold because if $t$ is an $i$-successor of $s$ for $i \in G$, then $s$ and $t$ agree on all formulas of the form $D_{H} \psi$ with $H \subseteq G$. CT2-CT3 hold because of step (c) and CT1 holds because of only retaining nodes marked satisfiable (check $(\mathrm{d}(\mathrm{B}))$. Note that one of the states $(p t$ successor of the root of the pre-tableau) contains $\phi$. By Lemma 7, $\phi$ is satisfiable.

For the other direction, we show that if $H M P(\phi)$ returns false, then $\phi$ is $C L D$-inconsistent and hence unsatisfiable. Exactly as in [16], it can be shown that if a node $s$ is not marked as satisfiable because it is either blatanly 
inconsistent or one of its $i$ successors is inconsistent, then $L(s)$ is $C L D$-inconsistent hence $\mathcal{C} \mathcal{L}$ unsatisfiable. For the case of one of $c t$ successors of $s$ being inconsistent, observe that this will make $L(s)$ inconsistent with respect to $C L$ axioms $\mathrm{G} 1$ and $\mathrm{G} 5$.

Hence, if the root of the pre-tableau is not marked satisfiable, then $\phi$ is unsatisfiable.

Lemma 10 It can be checked, using depth first search, in space polynomial in $|\phi|$, whether HMP( $\phi)$ will return true.

Proof The pre-tableau tree constructed by $\operatorname{HMP}(\phi)$ is (doubly) exponential in $|\phi|$, however we show, similarly to the proof in [16], that it is possible to check using only polynomial space whether $H M P(\phi)$ will return true. This is done by exploring the pre-tableau in a depth-first manner instead of constructing it as a complete tree.

In the proof of Lemma 8 we have shown that the depth of the tree is bounded by $O\left(|\phi|^{3}\right)$. A branch of this length can be stored using polynomial space, provided every search node also has size polynomial in $|\phi|$, and backtracking information (which branch to explore next) can also be represented using polynomial space.

Clearly, since the number of formulas in $L(s)$ is at most $|\phi|$, and the length of each formula is at most $|\phi|, L(s)$ can be represented in space polynomial in $|\phi|$.

To represent backtracking information, we need to be able to iterate through successors of a node using polynomial amount of space. There is a difficulty with $\mathcal{C L D}$ compared to pure epistemic logic, in that the branching factor of the pre-tableau is possibly exponential because of the number of $c t$-children of a node. However, an index of each such child requires polynomial space (if we number them from subset 1 to subset $3^{\mid} \phi \mid$, we can index them using $\log (3|\phi|)$ bits which is $O(|\phi|))$. The idea is that we generate an index of each next $c t$ successor one at a time and re-use the space to generate the next index when the check for the previous $c t$ successor returns true.

Given that the length of a path, hence the depth of the stack, is bounded by a polynomial in $|\phi|$, and the size of each search node on the stack is also bounded by a polynomial in $|\phi|$, the algorithm requires space polynomial in $|\phi|$.

This completes the proof that $\mathcal{C L D}$ satisfiability problem is PSPACE-complete.

\section{Adding Interaction Axioms}

The relationship between knowledge and ability has received substantial interest in the literature; see [1] for a recent overview. In particular, it has been noted that under imperfect information we can talk about several "levels" of ability [21, 18, 20], and that all of them are potentially interesting. For example, here are four different properties of an agent's ability to make $\phi$ come about, under the assumption that the agent has imperfect knowledge about the world:

1. The agent cannot make $\phi$ come about (there is no action the agent can perform that will ensure $\phi$ );

2. the agent is able to make $\phi$ come about, but she doesn't necessarily know it (there is an action the agent can perform that will ensure $\phi$, but that is not necessarily the case in other states the agent considers possible);

3. the agent is able to make $\phi$ come about and knows it, but does not necessarily know how (in every state the agent considers possible there is an action that will ensure $\phi$, but not necessarily the same action in each state);

4. the agent is able to make $\phi$ come about and knows how (there is an action that will make $\phi$ come about in every state the agent considers possible).

As discussed in the introduction, all of these different variants are potentially interesting, and it is important to be able to distinguish between them [21, 20]. Similar properties can be defined for groups of agents, using some notion of group knowledge such as distributed or common knowledge. The three first properties can intuitively be expressed in $\mathcal{E C L}$ as $\neg[i] \phi,[i] \phi$ and $K_{i}[i] \phi$, respectively. The fourth property, knowledge de re of the ability to make $\phi$ come about, cannot be expressed using standard combinations of epistemic and coalitional ability operators 
with standard semantics, and is also not even semantically definable in models which use effectivity functions rather than actions, as discussed in the introduction.

An advantage of the logics discussed so far, taking the second variant of ability mentioned above as a primary notion, is that it allows us to distinguish the second $([i] \phi)$ and third type $\left(K_{i}[i] \phi\right)$ in the same language. Still, it would be interesting to see what happens if we take the variant of ability in point 3 above as the primary notion - what is the logic of that notion of ability? As far as we know there are no existing completeness or complexity results taking the notion of de dicto knowledge-based ability as a primary notion.

In other words, we are interested in models where an agent (or a group) is able to make something come about if and only if they know that they can make it come about. Formally: $X \in E(s)(i)$ iff $\forall t$ such that $s \sim_{i} t$, $X \in E(t)(i)$ (note that the "if" direction holds immediately by reflexivity of the $\sim_{i}$ relation). This is equivalent to I1 in the following, where we also include corresponding interaction properties for groups:

I1 $s \sim_{i} t \Rightarrow E(s)(i)=E(t)(i)$

I2 $s \sim{ }_{G}^{C} t \Rightarrow E(s)(G)=E(t)(G)$

I3 $s \sim{ }_{G}^{D} t \Rightarrow E(s)(G)=E(t)(G)$

While I1 says that a single agent always knows what he can do, I2 and I3 say that a group always knows, either by distributed or common knowledge, what it can do.

These properties are captured by the following axioms, respectively (this claim will be made more precise in the following).

KAI $[i] \phi \rightarrow K_{i}[i] \phi$

KAC $[G] \phi \rightarrow C_{G}[G] \phi$

KAD $[G] \phi \rightarrow D_{G}[G] \phi$

Note that the implication in the other directon holds for all of these axioms, because a truth (T) axiom holds for each of these notions of knowledge. Thus, the intended notion of ability is captured: an agent or a group is able to make something come about if and only if they know it.

We now define the logics $\mathcal{C} \mathcal{L}{ }^{+}, \mathcal{C} \mathcal{L C}{ }^{+}$and $\mathcal{C} \mathcal{L} \mathcal{D}^{+}$. The languages of these logics are the languages of $\mathcal{C} \mathcal{L}$, $\mathcal{C} \mathcal{L C}$ and $\mathcal{C} \mathcal{L}$, respectively. The models of the logics are the classes of epistemic coalition models with the properties I1, I2 and I3, respectively. Interpretation is defined as before. We write $\models_{\mathcal{C} \mathcal{L} \mathcal{K}^{+}} \phi$ to mean that $\phi$ is valid on all $\mathcal{C} \mathcal{L} \mathcal{K}^{+}$models, and similarly for the other logics.

Let $C L K^{+}, C L C^{+}$and $C L D^{+}$be the result of extending the axiomatisations $C L K, C L C$ and $C L D$ with axioms KAI, KAC and KAD, respectively. The following is immediate.

Lemma 11 (Soundness) For any $\mathcal{C} \mathcal{L} \mathcal{K}$-formula $\phi, \vdash_{C L K^{+}} \phi$ implies $\models_{\mathcal{C} \mathcal{L} \mathcal{K}+} \phi$. For any $\mathcal{C} \mathcal{L} \mathcal{C}$-formula $\phi, \vdash_{C L C}$ $\phi$ implies $\models_{\mathcal{C} L \mathcal{C}^{+}} \phi$. For any $\mathcal{C} \mathcal{L} \mathcal{D}$-formula $\phi, \vdash_{C L D}+\phi$ implies $\left.\right|_{\mathcal{C L D}^{+}} \phi$.

Proof The proof is routine. We show the case for $C L D^{+}$: validity of the axiom KAD. Let $M, s \models[G] \phi$. This means that $E(s)(G) \subseteq \phi^{M}$. By I3, for all $t$ with $s \sim_{G}^{D} t, E(s)(G)=E(t)(G)$. Hence for all $t$ with $s \sim_{G}^{D} t$, $M, t=[G] \phi$. Hence $M, s \models D_{G}[G] \phi$.

We now prove completeness of two of these three logics, before we look at computational complexity.

\subsection{Completeness}

Theorem 8 Any $C L C^{+}$-consistent formula is satisfied in some model with the I2 property. 
Proof The proof is an adaption of the proof of Theorem 1 in Section 3. In the following we say "as before" to mean "as in the proof of Theorem 1".

The canonical playable model $M^{c}=\left(S^{c},\left\{\sim_{i}^{c}: i \in N\right\}, E^{c}, V^{c}\right)$ is defined exactly like in Theorem 1 . Let $\phi$ be a consistent formula. The closure $\operatorname{cl}(\phi)$ is also defined as before. We also use the abbreviation $[\psi]^{c}$ for $\left\{s \in S^{c}: \psi \in s\right\}$.

The filtration $M^{f}=\left(S^{f},\left\{\sim_{i}^{f}: i \in N\right\}, E^{f}, V^{f}\right)$ is constructed as follows (the only difference to the construction in the proof of Theorem 1 is the definition of $\left.E^{f}\right)$ :

$S^{f}$ is $\left\{[s]_{c l(\phi)}: s \in S^{c}\right\}$ where $[s]_{c l(\phi)}=s \cap \operatorname{cl}(\phi)$. We will omit the subscript $c l(\phi)$ in what follows for readability.

$[s] \sim_{i}^{f}[t]$ iff $\left\{\psi: K_{i} \psi \in[s]\right\}=\left\{\psi: K_{i} \psi \in[t]\right\}$.

$V^{f}([s])=\{p: p \in[s]\}$.

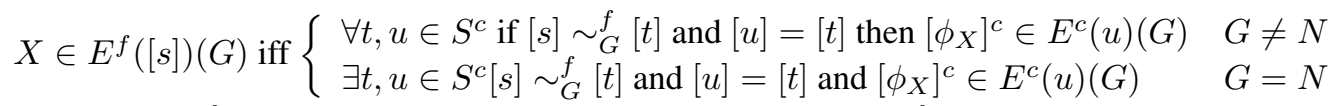

where $\sim_{G}^{f}$ is shorthand for the transitive closure of $\bigcup_{i \in G} \sim_{i}^{f}, \phi_{X}=\bigvee_{[t] \in X} \phi_{[t]}$ and $\phi_{[t]}$ is a conjunction of all formulas in $[t]$. To see that $E^{f}$ is well defined, observe that $[s]=\left[s^{\prime}\right] \Rightarrow E^{f}([s])(G)=E^{f}\left(\left[s^{\prime}\right]\right)(G)$ for any $G$.

In the following we use $\sim_{G}^{f}$ as shorthand for the transitive closure of $\bigcup_{i \in G} \sim_{i}^{f}$, as in the definition above.

We now prove by induction on the length of $\theta$ that for every $\theta \in \operatorname{cl}(\phi), M^{f},[s] \models \theta$ iff $\theta \in[s]$.

case $\theta=[G] \psi, G \neq N$

$M^{f},[s] \models[G] \psi$ iff $\psi^{M^{f}} \in E^{f}([s])(G)$ iff $\forall t, u \in S^{c}$ if $[s] \sim_{G}^{f}[t]$ and $[u]=[t]$ then $\left[\bigvee_{[t] \in \psi^{M^{f}}} \phi_{[t]}\right]^{c} \in$ $E^{c}(u)(G)$ iff (by the inductive hypothesis) $\forall t, u \in S^{c},[s] \sim_{G}^{f}[t]$ and $[u]=[t] \Rightarrow\left[\bigvee_{\psi \in[t]} \phi_{[t]}\right]^{c} \in$ $E^{c}(u)(G)$ iff (by Lemma 2.1) $\forall t, u \in S^{c},[s] \sim_{G}^{f}[t]$ and $[u]=[t] \Rightarrow[\psi]^{c} \in E^{c}(u)(G)$ iff (by Lemma 2.2) $\forall t, u \in S^{c},[s] \sim_{G}^{f}[t]$ and $[u]=[t] \Rightarrow[G] \psi \in u$.

We now show that $\forall t, u \in S^{c},[s] \sim_{G}^{f}[t]$ and $[u]=[t] \Rightarrow[G] \psi \in[u]$ holds iff $[G] \psi \in[s]$. The implication towards the right is immediate: take $t=u=s\left(\sim_{G}^{f}\right.$ is reflexive). For the other direction, let $t$ and $u$ be such that $[s] \sim_{G}^{f}[t]$ and $[u]=[t]$, and assume that $[G] \psi \in[s]$. We must show that $[G] \psi \in[u]$. By the definition of $\sim_{G}^{f}$, there are $\left[s_{1}\right], \ldots,\left[s_{k}\right]$ and $i_{1}, \ldots, i_{k-1}$ such that $\left[s_{1}\right] \sim_{i_{1}}^{f} \ldots \sim_{i_{k-1}}^{f}\left[s_{k}\right]$ and $[s]=\left[s_{1}\right]$ and $[t]=\left[s_{k}\right]$. We now show that for any $j, 1 \leq j \leq k-1,[G] \psi \in\left[s_{j}\right] \Rightarrow[G] \psi \in\left[s_{j+1}\right]$. Assume that $[G] \psi \in\left[s_{j}\right]$. By axiom KAC and the closure conditions, $C_{G}[G] \phi \in\left[s_{j}\right]$. By axiom $\mathrm{C}$ and the closure conditions, $K_{i_{j}} C_{G}[G] \psi \in\left[s_{j}\right]$. By definition of $\sim_{i_{j}}^{f}, K_{i_{j}} C_{G}[G] \psi \in\left[s_{j+1}\right]$. By the truth axiom (T) for $K_{i_{j}}$ and for $C_{G}$ (derivable), $[G] \psi \in\left[s_{j+1}\right]$. It follows that $[G] \psi \in[t]$, and thus that $[G] \psi \in[u]$ since $[G] \psi$ is in the closure.

case $\theta=[G] \psi, G=N$

$M^{f},[s] \models[G] \psi$ iff $\psi^{M^{f}} \in E^{f}([s])(G)$ iff $\exists t, u \in S^{c}[s] \sim_{G}^{f}[t]$ and $[u]=[t]$ and $\left[\bigvee_{[t] \in \psi^{M} f} \phi_{[t]}\right]^{c} \in$ $E^{c}(u)(G)$ iff (by the inductive hypothesis) $\exists t, u \in S^{c}[s] \sim_{G}^{f}[t]$ and $[u]=[t]$ and $\left[\bigvee_{\psi \in[t]} \phi_{[t]}\right]^{c} \in$ $E^{c}(u)(G)$ iff (by Lemma 2.1) $\exists t, u \in S^{c}[s] \sim_{G}^{f}[t]$ and $[u]=[t]$ and $[\psi]^{c} \in E^{c}(u)(G)$ iff (by Lemma 2.2) $\exists t, u \in S^{c}[s] \sim_{G}^{f}[t]$ and $[u]=[t]$ and $[G] \psi \in u$.

We now show that $\exists t, u \in S^{c}[s] \sim_{G}^{f}[t]$ and $[u]=[t]$ and $[G] \psi \in u$ holds iff $[G] \psi \in[s]$. The implication towards the left is immediate: take $t=u=s$. For the other direction, let $[s] \sim_{G}^{f}[t]$ and $[u]=[t]$ and $[G] \psi \in u$. We must show that $[G] \psi \in s$. By similar reasoning as in the $G=N$ case (and symmetry of the $\sim{ }_{G}^{f}$ relation, we get that $[G] \psi \in[t]$ and $[G] \psi \in[s]$.

All other cases Exactly as in the proof of Theorem 1. 


\section{Proposition $2 M^{f}$ satisfies E1-E5.}

\section{Proof}

E1 Again, recall that $\phi_{\emptyset}$ is the empty disjunction, $\perp$.

First, consider the case that $G \neq N . \emptyset \in E^{f}([s])(G)$ iff (by definition of $\left.E^{f}\right) \forall t, u \in S^{c}[s] \sim_{G}^{f}[t]$ and $[u]=$ $[t] \Rightarrow[\perp]^{c} \in E^{c}(u)(G)$ iff $\forall t, u \in S^{c}[s] \sim_{G}^{f}[t]$ and $[u]=[t] \Rightarrow \emptyset \in E^{c}(u)(G)$. Since $E^{c}$ satisfies E1, $\emptyset \notin E^{c}(s)(G)$, and since $\sim_{G}^{f}$ is reflexive, $\emptyset \notin E^{f}([s])(G)$.

Second, consider the case that $G=N . \emptyset \in E^{f}([s])(G)$ iff $\exists t, u \in S^{c}[s] \sim_{G}^{f}[t]$ and $[u]=[t]$ and $[\perp]^{c} \in E^{c}(u)(G)$ iff $\exists t, u \in S^{c}[s] \sim_{G}^{f}[t]$ and $[u]=[t]$ and $\emptyset \in E^{c}(u)(G)$. Since $E^{c}(u)$ satisfies E1 for every $u, \emptyset \notin E^{c}(u)(G)$ for every $u$, so $\emptyset \notin E^{f}([s])(G)$.

E2 First, consider the case that $G \neq N . S^{f} \in E^{f}([s])(G)$ iff $\forall t, u \in S^{c}[s] \sim_{G}^{f}[t]$ and $[u]=[t] \Rightarrow\left[\bigvee_{[t] \in S^{f}} \phi_{[t]}\right]^{c} \in$ $E^{c}(u)(G)$ iff $\forall t, u \in S^{c}[s] \sim_{G}^{f}[t]$ and $[u]=[t] \Rightarrow S^{c} \in E^{c}(u)(G)$. Since $E^{c}(u)$ satisfies E2 for every $u$, $S^{f} \in E^{f}([s])(G)$.

Second, consider the case that $G=N . S^{f} \in E^{f}([s])(G)$ iff $\exists t, u \in S^{c}[s] \sim_{G}^{f}[t]$ and $[u]=[t]$ and $\left[\bigvee_{[t] \phi_{[t]} \in S^{f}} \phi_{[t]}\right]^{c} \in E^{c}(u)(G)$ iff $\exists t, u \in S^{c}[s] \sim_{G}^{f}[t]$ and $[u]=[t]$ and $S^{c} \in E^{c}(u)(G)$. Since $E^{c}(s)$ satisfies E2, $S^{f} \in E^{f}([s])(G)$ (take $t=u=s$ ).

E3 Let $\bar{X} \notin E^{f}([s])(\emptyset)$. Then there is a $t$ and an $u$ such that $[s] \sim_{G}^{f}[t]$ and $[u]=[t]$ and $\left[\phi_{\bar{X}}\right]^{c} \notin E^{c}(u)(\emptyset)$. $\left[\phi_{\bar{X}}\right]^{c}$ is the complement of $\left[\phi_{X}\right]^{c}$, since $\phi_{\bar{X}}=\neg \phi_{X}$. Since $E^{c}(u)$ satisfies E3, this means that there are $t, u$ such that $[s] \sim_{G}^{f}[t],[u]=[t]$ and $\left[\phi_{X}\right]^{c} \in E^{c}(u)(N)$. Hence $X \in E^{f}([s])(N)$ by definition of $E^{f}([s])(G)$ in the case $G=N$.

E4 Let $X \subseteq Y \subseteq S^{f}$ and $X \in E^{f}([s])(G)$. As before, $\left.\left[\phi_{X}\right]^{c} \subseteq \phi_{Y}\right]^{c}$. First, consider the case that $G \neq N$. Since $X \in E^{f}([s])(G)$, we have $\left[\phi_{X}\right]^{c} \in E^{c}(u)(G)$ for every $t, u \in S^{c}$ such that $[s] \sim_{G}^{f}[t]$ and $[u]=[t]$. Since $E^{c}(u)$ satisfies E4, $\left[\phi_{Y}\right]^{c} \in E^{c}(u)(G)$ for every such $t$ and $u$, so $Y \in E^{f}([s])(G)$.

Second, consider the case that $G=N$. Since $X \in E^{f}([s])(G)$, we have $\left[\phi_{X}\right]^{c} \in E^{c}(u)(G)$ for some $t, u \in S^{c}$ such that $[s] \sim_{G}^{f}[t]$ and $[u]=[t]$. Since $E^{c}(u)$ satisfies E4, $\left[\phi_{Y}\right]^{c} \in E^{c}(u)(G)$ for some such $t, u$, so $Y \in E^{f}([s])(G)$.

E5 Let $X \in E^{f}([s])\left(G_{1}\right)$ and $Y \in E^{f}([s])\left(G_{2}\right)$ and $G_{1} \cap G_{2}=\emptyset$.

First, consider the case that $G_{1} \cup G_{2} \neq N$ : for every $t, u$ such that $[s] \sim_{G}^{f}[t]$ and $\left.[u]=[t], \phi_{X}\right]^{c} \in$ $E^{c}(u)\left(G_{1}\right)$ and $\left[\phi_{Y}\right]^{c} \in E^{c}(u)\left(G_{2}\right)$ and since $E^{c}(u)$ satisfies E5, $\left[\phi_{X}\right]^{c} \cap\left[\phi_{Y}\right]^{c} \in E^{c}(u)\left(G_{1} \cup G_{2}\right)$ for every such $t, u$. Again, note that $\left[\phi_{X}\right]^{c} \cap\left[\phi_{Y}\right]^{c}=\left[\bigvee_{[t] \in X \cap Y} \phi_{[t]}\right]^{c}$. Since for every $t, u$ such that $[s] \sim_{G}^{f}[t]$ and $[u]=[t]\left[\bigvee_{[t] \in X \cap Y} \phi_{[t]}\right]^{c} \in E^{c}(u)\left(G_{1} \cup G_{2}\right)$, we have that $X \cap Y \in E^{f}([s])\left(G_{1} \cup G_{2}\right)$.

Second, consider the case that $G_{1} \cup G_{2}=N$. We consider the three sub-cases:

$G_{1}=N, G_{2}=\emptyset$ : We have that there exist $t^{\prime}, u^{\prime}$ such that $[s] \sim_{G}^{f}\left[t^{\prime}\right]$ and $\left[u^{\prime}\right]=\left[t^{\prime}\right]$ and $\left[\phi_{X}\right]^{c} \in E^{c}\left(u^{\prime}\right)\left(G_{1}\right)$, and that for every $t, u$ such that $[s] \sim_{G}^{f}[t]$ and $[u]=[t],\left[\phi_{Y}\right]^{c} \in E^{c}(u)\left(G_{2}\right)$. Since $E^{c}\left(u^{\prime}\right)$ satisfies E5, $\left[\phi_{X}\right]^{c} \cap\left[\phi_{Y}\right]^{c} \in E^{c}\left(u^{\prime}\right)\left(G_{1} \cup G_{2}\right)$. By the same argument as in the $G_{1} \cup G_{2} \neq N$ case, that means that $\left[\bigvee_{[t] \in X \cap Y} \phi_{[t]}\right]^{c} \in E^{c}\left(u^{\prime}\right)\left(G_{1} \cup G_{2}\right)$, and since $G_{1} \cup G_{2}=N$ that means that $X \cap Y \in E^{f}([s])\left(G_{1} \cup G_{2}\right)$.

$G_{2}=N, G_{1}=\emptyset:$ Symmetric to the argument above.

$G_{1} \neq \emptyset, G_{2} \neq \emptyset, G_{1} \cup G_{2}=N$ : We have that for all $t, u$ such that $[s] \sim_{G}^{f}[t]$ and $[u]=[t],\left[\phi_{X}\right]^{c} \in$ $E^{c}(u)\left(G_{1}\right)$ and $\left[\phi_{Y}\right]^{c} \in E^{c}(u)\left(G_{2}\right)$. Take $t=u=s$. Since $E^{c}(s)$ satisfies E5, $\left[\phi_{X}\right]^{c} \cap\left[\phi_{Y}\right]^{c} \in$ $E^{c}(s)\left(G_{1} \cup G_{2}\right)$. By the same argument as above, $\left[\bigvee_{[t] \in X \cap Y} \phi_{[t]}\right]^{c} \in E^{c}(s)\left(G_{1} \cup G_{2}\right)$, and since $G_{1} \cup G_{2}=N$ that means that $X \cap Y \in E^{f}([s])\left(G_{1} \cup G_{2}\right)$. 
Finally, we must show the following.

Proposition $3 M^{f}$ satisfies I2.

Proof Let $[s] \sim_{G}^{f}[t]$. Let $X \in E^{f}([s])(G)$; we show that $X \in E^{f}([t])(G)$ (proof of inclusion in the other direction is symmetric).

Consider the case that $G \neq N$. Let $t^{\prime}, u \in S^{c},[t] \sim_{G}^{f}\left[t^{\prime}\right]$ and $[u]=\left[t^{\prime}\right]$. We must show that $\left[\phi_{X}\right]^{c} \in E^{c}(u)(G)$ (*). By transitivity of $\sim_{G}^{f}$ we have that $[s] \sim_{G}^{f}[t]$, and thus (*) follows from the fact that $X \in E^{f}([s])(G)$.

Consider the case that $G=N$. From $X \in E^{f}([s])(G)$ we have that there exist $t^{\prime}, u \in S^{c}$ such that $[s] \sim_{G}^{f}\left[t^{\prime}\right]$, $[u]=\left[t^{\prime}\right]$, and $\left[\phi_{X}\right]^{c} \in E^{c}(u)(G)$. From transitivity of $\sim_{G}^{f}$ we have that $[t] \sim_{G}^{f}\left[t^{\prime}\right]$, and it follows that also $X \in E^{f}([t])(G)$ because $\exists t^{\prime}, u \in S^{c}[t] \sim_{G}^{f}\left[t^{\prime}\right]$ and $[u]=\left[t^{\prime}\right]$ and $\left[\phi_{X}\right]^{c} \in E^{c}(u)(G)$.

This completes the proof of Theorem 8 , since $\phi$ belongs to one of the maximal consistent sets and hence is satisfied in the constructed model.

Corollary 5 (Completeness of $\left.C L C^{+}\right)$For any $\mathcal{C L C}$-formula $\phi$, $\models_{\mathcal{C} \mathcal{L C}^{+}} \phi$ implies $\vdash_{C L C^{+}} \phi$.

Corollary 6 (Completeness of $\left.C L K^{+}\right)$For any $\mathcal{C} \mathcal{L} \mathcal{K}_{\text {-formula }} \phi, \models_{\mathcal{C} \mathcal{L} \mathcal{K}^{+}} \phi \vdash_{C L K^{+}} \phi$.

Proof The proof is exactly like in the case of $C L C^{+}$, with the following modification: in the definition of $E^{f}$, let $\sim_{G}^{f}$ be $\sim_{i}^{f}$ when $G=\{i\}$ is a singleton, and the identity relation otherwise.

\subsection{Computational Complexity}

Theorem 9 The satisfiability problem for $\mathcal{C} \mathcal{L D}^{+}$is PSPACE-complete.

Proof We modify the definition of the closure $\operatorname{ccl}(\phi)$ of a formula $\phi$ from the proof of Theorem 7 by adding an extra condition:

$$
[G] \psi \in \operatorname{ccl}(\phi) \Rightarrow D_{G}[G] \psi \in \operatorname{ccl}(\phi)
$$

We add the following condition on $L(s)$ to the definition of a $\mathcal{C L D}$ tableau from the proof of Theorem 7:

ECT $[G] \psi \in L(s) \Rightarrow D_{G}[G] \psi \in L(s)$

Then we prove a lemma corresponding to Lemma 7 for $\mathcal{C} \mathcal{L D}^{+}$:

Lemma 12 An $\mathcal{C} \mathcal{L D}^{+}$formula $\phi$ is satisfiable if, and only if, there is a $\mathcal{C} \mathcal{L D}^{+}$tableau $T=\left(S, L, R_{1}, \ldots, R_{n}\right)$ for $\phi$.

Proof The direction from left to right (if there is a model for $\phi$, then there is a tableau for $\phi$ ) is straightforward.

As in the proof of Lemma 7, we construct a model $M=\left(S, E,\left\{\sim_{i}: i \in N\right\}, V\right)$ for $\phi$ given a tableau $T=\left(S, L, R_{1}, \ldots, R_{n}\right)$. The construction of $S, E,\left\{\sim_{i}: i \in N\right\}, V$ is exactly as before. We need to prove that

$$
\forall i \in G\left(s \sim_{i} t\right) \Rightarrow \forall X(X \in E(s)(G) \Leftrightarrow X \in E(t)(G))
$$

Let us assume that $s \sim_{i} t$. By the argument from the proof of Lemma 7, for all $\chi$ such that $D_{G} \chi \in L(s)$, $\chi \in L(t)$, and vice versa. Because of the ECT condition, $[G] \psi \in L(s)$ entails $D_{G}[G] \psi \in L(s)$, so for every $\psi$, $[G] \psi \in L(s)$ iff $[G] \psi \in L(t)$. In particular, $[N] \psi \in L(s)$ iff $[N] \psi \in L(t)$, and hence by CT1, $\neg[\emptyset] \neg \psi \in L(s)$ iff $\neg[\emptyset] \neg \psi \in L(t)$.

A simple inspection of the construction of $E(s)(G)$ for $G \neq N$ in the proof of Lemma 7 shows that if $s$ and $t$ agree on $[G] \psi$ formulas, then for every $X, X \in E(s)(G) \Leftrightarrow X \in E(t)(G)$. The case for $G=N$ follows because $s$ and $t$ agree on all formulas of the form $[\emptyset] \psi$. 
The $H M P(\phi)$ procedure needs to be modified as follows. Node $s$ can only be marked as satisfiable if for no $\psi$, $[G] \psi, \neg D_{G}[G] \psi \in L(s)$.

Since the closure and the node label still remain polynomial in the original formula $\phi$, the rest of the complexity proof goes through.

\section{Related Work}

In this section, we briefly survey related work, which falls in two categories: the work that introduces proof techniques for proving completeness and analysing complexity of logics of coalitional ability and for epistemic logics separately; and the work on combining logics of coalitional ability and epistemic logic. We mentioned some work on the interaction of knowledge and ability in the preceeding sections of the paper. For a survey of that work, see [1].

Coalition Logic was introduced by Pauly, and many of the proof techniques we used here originate from his paper [25]. Other logics of coalitional ability have also been extensively studied, for example Alternating-time Temporal Logic $(\mathcal{A T} \mathcal{L})$ [4], and STiT logics [5]. The techiques developed for those logics influence developments for $\mathcal{C} \mathcal{L}$ as well, especially since as observed in [10], $\mathcal{C} \mathcal{L}$ is a fragment of $\mathcal{A T} \mathcal{L}$. Most of the known meta-logical results for the logics of coalitional ability have been about computational complexity and expressive power. Completeness results have been harder to obtain, with Goranko's and van Drimmelen's completeness proof for $\mathcal{A T} \mathcal{L}$ [13], Pauly's completeness proof for $\mathcal{C} \mathcal{L}$ [25] and Broersen and colleagues' completeness proofs for different variants of STiT logic $[7,6,19]$ being notable exceptions.

There exists an even larger body of work on epistemic logic, both on axiomatisation and complexity, for example, [8, 22, 27]. Filtration techniques for group modalities have been studied extensively in [27], and tableau techniques introduced in [16] and developed for example in [2].

Epistemic extensions of coalitional ability have also been studied before. One of the most well known ones is $\mathcal{A} \mathcal{T} \mathcal{E}, \mathcal{A} \mathcal{T} \mathcal{L}$ extended with epistemic operators. In [28], some axioms of $\mathcal{A} \mathcal{T} \mathcal{E} \mathcal{L}$ are given, but there is no attempt to prove completeness. Complexity of of $\mathcal{A} \mathcal{T} \mathcal{E} \mathcal{L}$ was analysed in [30]. Guelev and colleagues [15, 14] proved completeness of a fragment of $\mathcal{A} \mathcal{T} \mathcal{L}$ with distributed knowledge operators and 'de re' knowledge of strategies. Broersen and colleagues $[6,19]$ proved completeness of variants of STiT logic that include individual knowledge operators, but not group knowledge operators. In [19] adding group knowledge operators is listed as an important challenge.

\section{Conclusions}

This papers settles several hitherto unsolved problems. It proves completeness of coalition logic extended with different combinations of group knowledge operators. The axioms for the epistemic modalities are standard in epistemic logic, but the completeness proofs require non-trivial combinations of techniques. The proofs are given in detail, and can be used and extended in future work. The paper furthermore completely characterises the computational complexity of the considered logics. They are all decidable. We can conclude that adding coalition operators to epistemic logic comes "for free" without changing the complexity of the satisfiability problem: the extension of epistemic logic with distributed and common knowledge with coalition operators remains EXPTIME-complete, the extension of epistemic logic with only distributed knowledge with coalition operators remains PSPACE-complete.

We studied two variants of coalitional ability in this paper. The first is standard ability, with no knowledge requirements, allowing us to distinguish between standard ability and knowledge of ability. By adding interaction axioms, we also obtained axiomatisations of another notion of ability, namely being able to make something come about if and only if you know that you can make it come about.

There are some open problems. We have not characterised the complexity of the satisfiability problem for $\mathcal{C} \mathcal{L C}^{+}$; we conjecture that it is EXPTIME-complete. We also have not proven completeness of $\mathcal{C} \mathcal{L D}^{+}$; we conjecture that the logic is complete. Completeness and complexity of logics with other interaction properties is also interesting for future work. 
Acknowledgements We thank the reviewers for their detailed and thorough comments that helped us to improve the paper.

\section{References}

[1] T. Ågotnes, V. Goranko, W. Jamroga, and M. Wooldridge. Knowledge and ability. In H. P. van Ditmarsch, J. Y. Halpern, W. van der Hoek, and B. P. Kooi, editors, Handbook of Logics for Knowledge and Belief. College Publications, London, 2015. Forthcoming.

[2] M. Ajspur, V. Goranko, and D. Shkatov. Tableau-based decision procedure for the multiagent epistemic logic with all coalitional operators for common and distributed knowledge. Logic Journal of the IGPL, 21(3):407437, 2013.

[3] N. Alechina, B. Logan, H. N. Nguyen, and A. Rakib. Logic for coalitions with bounded resources. Journal of Logic and Computation, 21(6):907-937, December 2011.

[4] R. Alur, T. A. Henzinger, and O. Kupferman. Alternating-time temporal logic. Journal of the ACM, 49:672713, 2002.

[5] N. Belnap and M. Perloff. Seeing to it that: a canonical form for agentives. Theoria, 54:175-199, 1988.

[6] J. Broersen. A complete STIT logic for knowledge and action, and some of its applications. In M. Baldoni, T. C. Son, M. B. van Riemsdijk, and M. Winikoff, editors, Declarative Agent Languages and Technologies VI, 6th International Workshop, DALT 2008, Estoril, Portugal, May 12, 2008, Revised Selected and Invited Papers, volume 5397 of Lecture Notes in Computer Science, pages 47-59, 2009.

[7] J. Broersen, A. Herzig, and N. Troquard. A normal simulation of coalition logic and an epistemic extension. In D. Samet, editor, Proceedings of the 11th Conference on Theoretical Aspects of Rationality and Knowledge (TARK-2007), Brussels, Belgium, June 25-27, 2007, pages 92-101, 2007.

[8] R. Fagin, J. Y. Halpern, Y. Moses, and M. Y. Vardi. Reasoning About Knowledge. The MIT Press, Cambridge, Massachusetts, 1995.

[9] D. M. Gabbay and V. B. Shehtman. Products of modal logics, part 1. Logic Journal of the IGPL, 6(1):73-146, 1998.

[10] V. Goranko. Coalition games and alternating temporal logics. In Proceeding of the Eighth Conference on Theoretical Aspects of Ra tionality and Knowledge (TARK VIII, pages 259-272. Morgan Kaufmann, 2001.

[11] V. Goranko, W. Jamroga, and P. Turrini. Strategic games and truly playable effectivity functions. Autonomous Agents and Multi-Agent Systems, 26:288-314, 2013.

[12] V. Goranko, W. Jamroga, and G. van Drimmelen. Axiomatic systems for alternating time temporal epistemic logics (extended abstract).

[13] V. Goranko and G. van Drimmelen. Complete axiomatization and decidability of alternating-time temporal logic. Theoretical Computer Science, 353(1):93-117, 2006.

[14] D. P. Guelev and C. Dima. Epistemic ATL with perfect recall, past and strategy contexts. In M. Fisher, L. van der Torre, M. Dastani, and G. Governatori, editors, Computational Logic in Multi-Agent Systems 13th International Workshop, CLIMA XIII, Montpellier, France, August 27-28, 2012. Proceedings, volume 7486 of Lecture Notes in Computer Science, pages 77-93. Springer, 2012.

[15] D. P. Guelev, C. Dima, and C. Enea. An alternating-time temporal logic with knowledge, perfect recall and past: axiomatisation and model-checking. Journal of Applied Non-Classical Logics, 21(1):93-131, 2011. 
[16] J. Y. Halpern and Y. Moses. A guide to completeness and complexity for modal logics of knowledge and belief. Artif. Intell., 54(2):319-379, 1992.

[17] D. Harel, D. Kozen, and J. Tiuryn. Dynamic Logic. MIT Press, 2000.

[18] A. Herzig and N. Troquard. Knowing how to play: Uniform choices in logics of agency. In Proceedings of AAMAS'06, pages 209-216, 2006.

[19] A. H. J. Broersen and N. Troquard. What groups do, can do, and know they can do: an analysis in normal modal logics. Journal of Applied Non-Classical Logic, 19(3):261-289, 2009.

[20] W. Jamroga and T. Ågotnes. Constructive knowledge: what agents can achieve under imperfect information. Journal of Applied Non-Classical Logics, 17(4):423-475, 2007.

[21] W. Jamroga and W. van der Hoek. Agents that know how to play. Fundamenta Informaticae, 63:185-219, 2004.

[22] J.-J. C. Meyer and W. van der Hoek. Epistemic Logic for AI and Computer Science. Cambridge University Press: Cambridge, England, 1995.

[23] J. W. Moon and L. Moser. On cliques in graphs. Israel Journal of Mathematics, 3:23-28, 1965.

[24] H. N. Nguyen. Reasoning about resource-bounded multi-agent systems. PhD thesis, University of Nottingham, 2011.

[25] M. Pauly. A modal logic for coalitional power in games. Journal of Logic and Computation, 12(1):149-166, 2002.

[26] P. Y. Schobbens. Alternating-time logic with imperfect recall. Electronic Notes in Theoretical Computer Science, 85(2), 2004.

[27] W. van der Hoek and J.-J. C. Meyer. A complete epistemic logic for multiple agents combining distributed and common knowledge. In M. Bacharach, L. Gerard-Varet, P. Mongin, and H. Shin, editors, Epistemic Logic and the Theory of Games and Decisions, pages 35-68. Kluwer Academic Publishers, Dordrecht, 1997.

[28] W. van der Hoek and M. Wooldridge. Cooperation, knowledge and time: Alternating-time temporal epistemic logic and its applications. Studia Logica, 75:125-157, 2003.

[29] H. van Ditmarsch, W. van der Hoek, and B. Kooi. Dynamic Epistemic Logic, volume 337 of Synthese Library. Springer, 2007.

[30] D. Walther. ATEL with common and distributed knowledge is ExpTime-complete. In Proceedings of M4M-4: the 4th Workshop on Methods for Modalities, 2005.

[31] Y. Wang and T. Ågotnes. Public announcement logic with distributed knowledge. In H. van Ditmarsch, J. Lang, and S. Ju, editors, Third International Workshop, LORI 2011, Guangzhou, China, October 10-13, 2011. Proceedings, volume 6953 of Lecture Notes in Computer Science/Lecture Notes in Artificial Intelligence. Springer, 2011. 